\title{
The Left Inferior Frontal Gyrus: A neural crossroads between abstract and concrete knowledge
}

Pasquale Anthony Della Rosa ${ }^{1 *}$, Eleonora Catricalà ${ }^{2 *}$, Matteo Canini ${ }^{1}$, Gabriella Vigliocco ${ }^{3}$, Stefano F. Cappa ${ }^{2,4}$

*equal contribution

Affiliations:

${ }^{1}$ Unit of Neuroradiology, IRCCS Ospedale San Raffaele, Milan, Italy

${ }^{2}$ NETS, Center for Neurocognition, Epistemology and Theoretical Syntax, Institute for Advanced Study-Pavia, Pavia, Italy

${ }^{3}$ Department of Experimental Psychology, University College London, London, UK

${ }^{4}$ IRCCS S. Giovanni di Dio Fatebenefratelli, Brescia, Italy

Corresponding Author: Pasquale Anthony Della Rosa

e-mail: pasqualeanthony.dellarosa@gmail.com; Mail address: Via Olgettina 60, 20132 Milan, Italy; Phone: +3902 2643 3634, Fax: +3902 26433447 


\begin{abstract}
Evidence from both neuropsychology and neuroimaging suggests that different types of information are necessary for representing and processing concrete and abstract word meanings. Both abstract and concrete concepts, however, conjointly rely on perceptual, verbal and contextual knowledge, with abstract concepts characterized by low values of imageability (IMG) (low sensory-motor grounding) and low context availability (CA) (more difficult to contextualize). Imaging studies supporting differences between abstract and concrete concepts show a greater recruitment of the left inferior frontal gyrus (LIFG) for abstract concepts, which has been attributed either to the representation of abstract-specific semantic knowledge or to the request for more executive control than in the case of concrete concepts. We conducted an fMRI study on 27 participants, using a lexical decision task involving both abstract and concrete words, whose IMG and CA values were explicitly modelled in the separate parametric analyses. The LIFG was significantly more activated for abstract than for concrete words, and a conjunction analysis showed a common activation for words with low IMG or low CA only in the LIFG, in the same area reported for abstract words. A regional template map of brain activations was then traced for words with low IMG or low CA, and BOLD regional time-series were extracted and correlated with the specific LIFG neural activity elicited for abstract words. The regions associated to low IMG, which were functionally correlated with LIFG, were mainly in the left hemisphere, while those associated with low CA were in the right hemisphere. Finally, in order to reveal which LIFG-related network increased its connectivity with decreases of IMG or CA, we conducted generalized psychophysiological interaction analyses. The connectivity strength values extracted from each region connected with the LIFG were correlated with specific LIFG neural activity for abstract words, and a regression analysis was conducted to highlight which areas recruited by low IMG or low CA predicted the greater activation of the IFG for abstract concepts. Only the left middle temporal gyrus/angular gyrus, known to be involved in semantic processing, was a significant predictor of LIFG activity differentiating abstract from concrete words.
\end{abstract}

The results show that the abstract conceptual processing requires the interplay of multiple brain regions, necessary for both the intrinsic and extrinsic properties of abstract knowledge. The LIFG can be thus identified as the neural crossroads between different types of information equally necessary for representing processing and differentiating abstract concepts from concrete ones. 
Keywords: abstract concepts, concrete concepts, imageability, context availability, inferior frontal gyrus, generalized psychophysiological interaction analyses

\section{Introduction}

When we think about a "dog" we will generally refer to it as being "concrete" on the basis of a great amount of external sensorial information. When we think about "justice", instead, we generally refer to internal sensory experience and linguistic information, which we define as being "abstract" (Vigliocco et al., 2009, 2013; Kousta et al., 2011). Both concrete and abstract word meanings, however, may rely jointly, despite differently, on all these kinds of information. The way in which we rely on different types of information for representing a dog (i.e., to bark, love for animals, is domestic) and justice (i.e., prison, law, to lie) still needs to be clarified.

According to Dual Coding Theory (Paivio, 1991) concrete concepts are supported by both perceptual and verbal knowledge, while abstract concepts rely only on verbal information. In contrast, the context availability theory (Schwanenflugel and Shoben, 1983) proposes that differences are due to the quantity and availability of contextual information, which is more readily available for concrete than abstract words.

These theoretical accounts have been translated operationally respectively in two constructs: imageability (IMG), which measures the difficulty/ease with which a word evokes a mental image (Paivio et al., 1968) and context availability (CA), which measures the difficulty/easy to think of a context for a word. Higher values of IMG are associated to sensory-motor information, mainly characterizing concrete words, while the meaning of abstract words is mediated mainly through language, resulting in a low value of IMG. In addition, it is generally easier to find a context for concrete words, while it is harder to generate a plausible context in which an abstract word can be represented (Schwanenflugel, Harnishfeger, \& Stowe, 1988; Schwanenflugel \& Shoben, 1983).

Under the assumptions of both Dual Code Theory and Context Availability Theory, imageability is defined as a semantic property of word representation. Both concrete and abstract word meanings are also bound to context, even though abstract words have less sensory context and should therefore be more reliant on the internal and linguistic contexts in which they appear.

If imageability can be assumed as an intrinsic semantic property, the difference between low and high imageable words depends on how the semantic representations are characterized within the semantic system (Paivio, 1986, 2010). If context availability can be thought instead as an extrinsic property of word meaning, the difference between concrete and abstract words will be influenced by differences in the way these representations depend more or less on context. 
Imaging studies supporting differences between abstract and concrete words show, quite consistently, a greater activation of the left inferior frontal gyrus (IFG) for abstract than concrete concepts (Perani et al., 1999; Binder et al., 2005; Noppeney \& Price, 2004; Fiebach \& Friederici, 2004; Friederici et al., 2000; Jessen et al., 2000), see table 1. Abstract concepts have been differently and interchangeably characterized on the basis of concreteness, imageability or context availability.

Table 1: Characteristics of the imaging studies reporting a greater involvement of left IFG for abstract than concrete words.

\begin{tabular}{|c|c|c|c|c|c|c|c|}
\hline & $\begin{array}{l}\text { Definition of } \\
\text { abstract stimuli }\end{array}$ & $\begin{array}{l}\text { Other relevant } \\
\text { variables }\end{array}$ & $\begin{array}{l}\text { Stimuli } \\
\text { Matching }\end{array}$ & Rts & Task & $\begin{array}{l}\text { Involvement of the } \\
\text { left IFG }\end{array}$ & $\begin{array}{l}\text { hypothesized } \\
\text { IFG role }\end{array}$ \\
\hline $\begin{array}{l}\text { Hoffman et } \\
\text { al., } 2015\end{array}$ & $\begin{array}{l}\text { Imageability } \\
\text { ABS probe: } 295 \\
\text { (220-429) } \\
\text { CNC probe: } 581 \\
\text { (497-655) } \\
\text { ABS choice: } \\
300 \text { (210-503) } \\
\text { CNC choice: } \\
572 \text { (408-652) }\end{array}$ & $\begin{array}{l}\text { Concreteness: } \\
A B S<C N C \\
\text { semantic } \\
\text { diversity: } \\
A B S>C N C\end{array}$ & $\begin{array}{l}\text { Length and } \\
\text { frequency } \\
\text { (only for } \\
\text { probes): } \\
\text { ABS=CNC } \\
\text { Familiarity: } \\
\text { ABS<CNC } \\
\text { Age of } \\
\text { acquisition: } \\
\text { ABS }>C N C\end{array}$ & $A B S>C N C$ & $\begin{array}{l}\text { synonym } \\
\text { judgement task; } \\
\text { conditions: } \\
\text { relevant and } \\
\text { irrelevant cue }\end{array}$ & $\begin{array}{l}-A B S>C N C \\
\text {-irrelevant>relevant } \\
\text {-No interaction } \\
\text { between type of } \\
\text { stimuli and condition }\end{array}$ & $\begin{array}{l}\text { Semantic } \\
\text { control }\end{array}$ \\
\hline $\begin{array}{l}\text { Binder et al., } \\
2005\end{array}$ & & $\begin{array}{l}\text { Imageability } \\
\text { CNC: 604(577- } \\
644) \\
\text { ABS: 328(233- } \\
370) \\
\text { Concreteness } \\
\text { CNC:586(421- } \\
662) \\
\text { ABS:310(217- } \\
436)\end{array}$ & $\begin{array}{l}\text { Letter } \\
\text { length, } \\
\text { phonemes, } \\
M P B F, O N \text { : } \\
A B S=C N C\end{array}$ & $A B S>C N C$ & $\begin{array}{l}\text { Lexical decision } \\
\text { task }\end{array}$ & $A B S>C N C$ & $\begin{array}{l}\text { phonological } \\
\text { working } \\
\text { memory } \\
\text { and lexical } \\
\text { retrieval }\end{array}$ \\
\hline $\begin{array}{l}\text { Noppeney \& } \\
\text { Price, } 2004\end{array}$ & Not specified & / & $\begin{array}{l}\text { Frequency } \\
\text { and number } \\
\text { of } \\
\text { letters } \\
\text { syllables: } \\
\text { ABS=CNC }\end{array}$ & $A B S=C N C$ & $\begin{array}{l}\text { Semantic } \\
\text { similarity } \\
\text { decision: easy } \\
\text { and difficult }\end{array}$ & $\begin{array}{l}\text {-ABS > sensory-based } \\
\text { knowledge (visual, } \\
\text { sounds, actions) } \\
\text {-easy ABS } \\
\text { trials > difficult } \\
\text { sensory based } \\
\text { knowledge }\end{array}$ & $\begin{array}{l}\text { retrieval } \\
\text { mechanism or } \\
\text { strategy for } \\
\text { accessing } \\
\text { abstract } \\
\text { semantics }\end{array}$ \\
\hline $\begin{array}{l}\text { Sabsevitz et } \\
\text { al., } 2005\end{array}$ & I & $\begin{array}{l}\text { Imageability: } \\
A B S<C N C\end{array}$ & $\begin{array}{l}\text { Letter } \\
\text { length: } \\
A B S=C N C\end{array}$ & $A B S=C N C$ & $\begin{array}{l}\text { Semantic } \\
\text { similarity } \\
\text { decision: easy } \\
\text { and difficult } \\
\end{array}$ & $A B S>C N C$ & $\begin{array}{l}\text { Verbal } \\
\text { semantic } \\
\text { system }\end{array}$ \\
\hline $\begin{array}{l}\text { Fiebach \& } \\
\text { Friederici, } \\
2003\end{array}$ & $\begin{array}{l}\text { Concreteness } \\
\text { ABS: } 1.95 \\
(0.09) \\
\text { CNC: } 3.55 \\
(0.08) \\
\end{array}$ & / & $\begin{array}{l}\text { Frequency: } \\
A B S=C N C\end{array}$ & $A B S=C N C$ & $\begin{array}{l}\text { Lexical decision } \\
\text { task }\end{array}$ & $A B S>C N C$ & $\begin{array}{l}\text { controlled } \\
\text { semantic } \\
\text { retrieval }\end{array}$ \\
\hline $\begin{array}{l}\text { Perani et al., } \\
1999\end{array}$ & I & / & $\begin{array}{l}\text { Length and } \\
\text { number of } \\
\text { syllables: } \\
A B S=C N C\end{array}$ & $A B S=C N C$ & $\begin{array}{l}\text { Lexical decision } \\
\text { task }\end{array}$ & $A B S>C N C$ & Not specified \\
\hline
\end{tabular}

This consistent finding has been interpreted in different ways. Following the intimate link between language and abstract word representation postulated in the dual code theory, some researchers interpreted the IFG activation as reflecting the involvement in a verbal semantic network supporting abstract representations (Sabsevitz et al., 2005; Binder et al., 2009). In agreement with the context availability theory, the greater activation of the IFG for abstract concepts has been attributed to the 
greater executive control demands exerted by these stimuli, as their meanings depend more upon context and consequently require more regulation than concrete words (Hoffman et al., 2015; Fiebach and Friederici, 2004).

In this study, we aimed at unravelling the role of the left inferior frontal gyrus in the representation and processing of abstract concepts, in particular on the basis of different connections driven by different types of information (i.e., IMG and CA), all contributing in abstract concepts' representations and processing.

On the basis of previous evidence, we expect an involvement of the left IFG for abstract concepts more than concrete concepts (classified on the basis of concreteness scale), for less imageable words and for words with a lower context availability rating score. However, imageability and context availability tap into at least partially different aspects of semantic representations and processing, according to the above-mentioned theoretical accounts. We thus predicted that imageability and context availability may contribute differently to the representation and processing of abstract and concrete concepts, representing respectively their intrinsic and extrinsic underlying properties.

The LIFG region is engaged by multiple cognitive tasks (Liakakis et al., 2011), and its activation may represent the shared tip of an iceberg, appearing in many studies but subtended by partially different functional activity patterns. In this case, we hypothesize that the characteristics of the intrinsic and extrinsic information (IMG and/or CA) necessary to represent and process word meaning may engage the LIFG differently.

Our main aim was to unravel the role of the IFG on the basis of different functional associations and connections driven by selected aspects of abstract representations (i.e., IMG and CA) in the context of a lexical decision task.

Translated in neural terms, first we want to observe which of the areas modulated by information pertaining to each of the two variables (i.e. the pattern of areas which create the informational basis for IMG and CA) is functionally correlated with brain activity in the area which responds significantly more for the distinction between what is abstract with respect to what is concrete. The assumption is that the information grounded in such areas is 'indirectly' responsive and tied to what we represent and categorize as abstract rather than concrete at a semantically higher hierarchical level. We used functional connectivity assuming that interregional correlations between LIFG abstract>concrete activity and different components of the IMG and CA circuits can detect a multimodal common informational grounding for "the representation of abstractness". 
Our second objective was to establish in which areas sensitive to the information contained in the IMG or the CA parameter the BOLD signal is modulated by the "direct influence" of the IMG or CA on the BOLD signal in LIFG related to abstractness and necessary for processing abstract knowledge in a lexical decision task. We thus used an independent whole-brain psychophysiological interaction (PPI) analysis to assess context-dependent changes in the direct influence of one brain region on another.

\section{Materials \& Methods}

\section{Subjects}

27 right-handed native Italian speakers (mean age $=23.3 \pm 2.60$ years (range: $20-29$ years) (8 males, 19 females) with normal hearing and vision, no history of neurological or psychiatric illness, and no early exposure to a second language participated in the study. All provided written informed consent. The study complied with all provisions of the Declaration of Helsinki and was approved by the San Raffaele Hospital Ethics Committee.

\section{Stimuli}

Thirty-five abstract (ABS) and 35 concrete (CNC) nouns were selected from Della Rosa et al. database (2011) on the basis of the median of the concreteness scale distribution (ABS mean = 331,34; $\mathrm{CNC}$ mean $=590,83 ; \mathrm{t}=17.27 \mathrm{p}<.0001)$, in order to be representative stimuli. We also verified that both imageability $(\mathrm{t}=12.23 \mathrm{p}<.0001)$ and context availability $(\mathrm{t}=5.59 \mathrm{p}<.0001)$ resulted significantly different. Abstract and concrete words were instead matched for other psycholinguistic variables known to affect abstract and concrete concepts processing, i.e. Mode of Acquistion $(\mathrm{t}=$ $.037 \mathrm{p}=.97)$ (Della Rosa et al., 2011), Emotional Valence $(\mathrm{t}=-.238 \mathrm{p}=.81)$ (Kousta et al., 2011; Vigliocco et al., 2013); Age of Acquisition ( $\mathrm{t}=-.267 \mathrm{p}=.79)$ (Cortese $\&$ Khanna; 2007), for number of letters $(\mathrm{t}=-.268 \mathrm{p}=.789)$, but not for familiarity $(\mathrm{t}=3.97 \mathrm{p}<.0001)$, see table 2 .

Table 2: Values of the variables of interest of abstract and concrete stimuli.

\begin{tabular}{|c|c|c|c|c|c|c|c|c|c|c|}
\hline abstract / concrete & & $\mathrm{CNC}$ & IMG & CA & FAM & AoA & MoA & Nlett & arousal & valence \\
\hline \multirow{4}{*}{ abstract $(n=35)$} & mean & 331,35 & 380,27 & 481,65 & 555,57 & 338,61 & 409,79 & 7,69 & 5,99 & 5,28 \\
\hline & St. dev. & 76,85 & 87,50 & 67,30 & 58,09 & 70,89 & 71,41 & 1,68 & 1,46 & 2,37 \\
\hline & Min. & 136,36 & 208,82 & 338,24 & 436,84 & 191,67 & 280,00 & 5,00 & 2,95 & 1,33 \\
\hline & Max. & 468,18 & 620,00 & 597,06 & 651,52 & 479,17 & 577,42 & 12,00 & 8,56 & 9,00 \\
\hline \multirow{2}{*}{ concrete $(n=35)$} & mean & 590,77 & 599,77 & 566,42 & 473,54 & 333,40 & 409,04 & 7,57 & 5,00 & 5,17 \\
\hline & St. dev. & 44,64 & 60,12 & 59,18 & 107,56 & 92,00 & 72,74 & 1,88 & 1,00 & 1,37 \\
\hline
\end{tabular}




\begin{tabular}{c|rrrrrrrrrr} 
Min. & 500,00 & 467,65 & 455,00 & 320,59 & 145,45 & 280,00 & 5,00 & 3,58 & 1,89 \\
\hline Max. & 684,21 & 697,06 & 695,65 & 691,18 & 515,00 & 594,74 & 13,00 & 6,89 & 8,37 \\
\hline
\end{tabular}

CNC: concreteness; IMG: imageability; CA: Context Avalaibility; FAM: Familiarity; AoA: Age of Acquisition; MoA: Mode of Acquisition; Nlett: number of letter.

A total of 70 orthographically legal pseudowords were constructed from the word stimuli by randomly exchanging one or two letters (but not the initial letter) (Fiebach et al., 2002) with Random Word Generator (https://www.gammadyne.com/rndword.htm), which can extrapolate random pseudowords from a given word. The deviance from the original word was set to a maximum of two letters, randomly picked among consonants and vowels at any site within the word, excluding the initial and final letter. Alternative pseudowords were generated for each of the word stimuli and one phonologically plausible candidate was selected.

\section{Experimental procedure}

A standard lexical decision task was used. All stimuli were white text (font size $=34 \mathrm{pt}$ ) centered on a black background. Each trial consisted of a lowercase word, a lowercase pseudo-word or a null (fixation) event presented for $1000 \mathrm{msec}$, followed by a 3, 5 or 6 second jittered interstimulus interval (ISI) (mean ISI $=4.241 \mathrm{msec}$ ), where a blank screen was presented during which participants were instructed to decide as quickly and as accurately as possible whether the stimulus was a word or not by finger-pressing one of two buttons on a response box.

A pseudo-randomized event-related fMRI paradigm was optimized for a total of 140 events (70 words and 70 psuedowords) and additional null-events. Trial order was pseudo-randomized so as to not contain more than three consecutive trials of a given condition (i.e. word,or pseudoword), The order of abstract and concrete stimuli in the word and pseudoword conditions was fixed through the alternation of word-word or word-pseudoword combinations and the frequency of null (fixation) events was determined, which accounted for approximately $1 / 3$ of trials, both in order to maximize the efficiency of the event-related design (Dale, 1999). The presentation order of stimuli was counterbalanced across participants, according to four possibilities provided by the optimization procedure. The total duration of a fMRI run was $8 \mathrm{~min} 51 \mathrm{~s}$. All events were evaluated during two functional runs in one fMRI session.

Inside the scanner, stimuli were presented through Presentation software (NeuroBehavioral Systems Inc., Albany, CA) via a PC placed outside the magnet room. A projector delivered stimuli on a translucent screen placed at the foot of the magnet bore. Participants viewed the screen through a mirror system attached to the top of the head coil. 
Prior to fMRI scanning, each subject read the instructions for the task and subsequently performed a small training session, consisting in 20 trials (10 words and 10 psuedowords) not included in the experiment, in order to familiarize with the task.

\section{fMRI acquisition, preprocessing and analysis fMRI Data Acquisition}

An fMRI event-related technique was used (3T Intera Philips body scanner, Philips Medical Systems, Best, NL, 8 channels-sense head coil, sense reduction factor $=2$, TE $=30 \mathrm{~ms}, \mathrm{TR}=2000$ $\mathrm{ms}, \mathrm{FOV}=240 \times 240$, matrix size $=96 \times 96,38$ axial slices per volume, 258 volumes for each run, slice thickness $=3 \mathrm{~mm}$ ). Furthermore, optimal EPI parameters at 3T were defined in order to gain BOLD sensitivity in the frontal and temporal cortices (Weiskopf et al., 2006). Specifically, in order to minimize susceptibility induced artefacts and signal dropouts, the slice tilt was set to 20 degrees on the (RL) tangent. The phase encoding (PE) gradient polarity was chosen to be negative with the phase encoding direction going form the anterior part to the posterior part of the brain. Five dummy scans preceded each run, all of which were then discarded prior to data analysis to optimize EPI image signal. For each subject a high-resolution structural image was acquired for means of coregistration, segmentation and spatial normalisation of the EPI scans (MPRAGE, 150 slice T1-weighted image, $\mathrm{TR}=8.03 \mathrm{~ms}, \mathrm{TE}=4.1 \mathrm{~ms}$; flip angle $=8^{\circ}, \mathrm{TA}=4.8 \mathrm{~min}$, resolution $\left.=1 \mathrm{~mm} \times 1 \mathrm{~mm} \times 1 \mathrm{~mm}\right)$ in the axial plane.

\section{fMRI Data Preprocessing}

Image preprocessing was performed using SPM8 (http://www.fil.ion.ucl.ac.uk/spm). Data preprocessing for each subject included: 1) EPI time-series diagnostics using tsdiffana (Matthew Brett, MRC CBU, http://imaging.mri-cbu.cam.ac.uk/imaging/DataDiagnostics), 2) alignment and orientation of structural images to improve segmentation accuracy, 3) co-registration of the EPI scans to the structural volume, 4) T1-weighted image tissue segmentation using the 'new segment' tool in SPM8 and generation of deskulled bias-corrected T1 images, 5) study-specific template creation using diffeomorphic image registration (DARTEL) in SPM8 and subject-specific flow fields generation containing the spatial deformations to normalize the EPI images into a common MNI coordinate space, 6) co-registered EPI time-series noise filtering (ArtRepair toolbox: http://cibsr.stanford.edu/tools/ArtRepair/ArtRepair.htm), motion and distortion correction using subject-specific field-map parameters (realign and unwarp, SPM8) and suppression of residual motion effects with Art Repair toolbox, 7) creation of a deskulled mean functional mask to remove 
nonbrain tissue from co-registered, noise-, motion-, and distortion-corrected EPI time-series in order to increase sharpness and avoid mismatch between alignment of the EPI data to the T1 image, 8) affine normalization of EPI data to MNI space with DARTEL flow fields, according to smooth deformations for each subject's native space gray, 9) spatial smoothing using a Gaussian kernel of 6 $\mathrm{mm}$.

\section{fMRI Data Analysis}

The final sample included in fMRI data analysis consisted of 26 subjects. Data from one subject was excluded due to low and abnormal behavioral performance on the lexical decision task.

Supplementary Figure S1 presents a temporal signal-to-noise ratio average map, showing EPI image quality over the whole brain.

The following first-level General Linear Model (GLM) analyses were designed 1) to trace the categorical difference, in terms of increased brain activity, between abstract and concrete words; 2) to identify which type of semantic information (imageability and context availability) and how the semantic weight of each word, defined respectively in terms of higher and lower values of imageability or context availability, can contribute to differentiate between abstract and concrete words.

1) We constructed an "abstractlconcrete dichotomic" model that included two trial types - word responses and pseudoword responses. Three parametric modulators were then included for word responses - one coding RT, one for accuracy ( 0 for incorrect responses if any) and the other abstractlconcrete status ( 1 for abstract words and 0 for concrete words). In this model, processing effort for words will be captured by the RT parametric modulator, whereas the abstractlconcrete status categorical modulator will capture differential engagement for abstract vs. concrete words, independent of RT.

2) a. We constructed an "imageability" model that included two trial types — word responses and pseudoword responses. Three parametric modulators were then included for word responses —one coding RT, one for accuracy ( 0 for incorrect responses if any) and the other including imageability ratings for each word (Paivio, 1971, 1986; Della Rosa et al., 2010). In this model, processing effort for words will be captured by the RT parametric modulator, whereas the imageability parametric modulator will capture brain activity coupled with increases or decreases of sensory-based perceptual representations (Sabsevitz et al., 2005), independent of RT.

2) b. We constructed a "context-availability" model that included two trial types — word responses and pseudoword responses. Three parametric modulators were then included for word responses - 
one coding RT, one for accuracy ( 0 for incorrect responses if any) and the other including context availability ratings for each word (Schwanenflugel \& Stowe, 1989; Della Rosa et al., 2011). In this model, processing effort for words will be captured by the RT parametric modulator, whereas the context availability parametric modulator will capture brain activity associated to words with a greater or smaller quantity of information (i.e. stronger or weaker links to contextual information stored in semantic memory) (Sabsevitz et al., 2005), independent of RT.

The default serial orthogonalisation procedures in the SPM software ensured that the RT (first) parametric modulator captures any shared activation between RT and the abstract status (Model 1), or imageability (Model 2) or context availability (Model 3) modulator, allowing to test for neural engagement differences due to abstract status, type and amount of information over and above effects of processing effort (RT).

In each model, events were convolved with the SPM12 canonical hemodynamic response function (HRF). Low frequency signal drifts were removed with a high-pass filter (128 s) and AR1 correction for serial autocorrelation was applied.

\section{The representation of abstractness}

The logical flow and all the methodological steps performed for this section are illustrated and detailed in Supplementary Figure 2.

First, one-sample t-tests in SPM were used on contrast images for second level group analyses using participants as a random effect, a) to identify brain regions that consistently responded to the abstract status of words (Model 1), b) to isolate brain activity engaged with increasing and decreasing imageability (Model 2) or context availability (Model 3). Statistical maps for model 1, 2 and 3 were family-wise error (FWE) corrected using threshold free cluster enhancement (TFCE) (Smith and Nichols, 2009). Significance and its correction for multiple comparisons is determined via permutation testing so that clusters are determined without arbitrary height thresholds (i.e. "threshold free"). The TFCE analysis was implemented with the TFCE toolbox in SPM12. We performed 1000 permutations and set the significance threshold to FWE $\mathrm{p}=0.1$ corrected TFCE analysis.

Second, we elucidated the functional association between imageability and context availability regions (i.e. clusters of activation for IMG and CA increases and decreases assessed at $\mathrm{p}<.005, \mathrm{k}>10$ 
voxels resulting respectively from Model 2 and Model 3) with LIFG activation cluster for abstract words, highlighted in Model 1.

In order to highlight which brain areas specific of IMG and CA is significantly and specifically related to LIFG activity for representing and processing abstractness we investigated the pattern of activation in statistical maps at a more lenient threshold (0.005 uncorrected voxelwise, cluster extent $=10$ voxels). This of course increases the chance of false positives, and the results should be interpreted with caution, exclusively in relation to previous findings reporting differences between abstract and concrete concepts in brain regions known to be implicated in both semantic representation and processing of word meanings. We decided a priori to use a $\mathrm{p}<.005$ uncorrected voxelwise value to create a very lenient filter as a first step for the interregional correlational analysis, whose aim was making inference upon areas surviving p-values corrected with bootstrapping methods. It must be underlined that the fMRI thresholding used to select clusters as input to the interregional correlational analysis was set only for purposes of region selection, in order to avoid the risk of neglecting subtle effects driven by either one of the parameters in any brain area related to the abstract/concrete distinction captured by LIFG activity. For this specific reason all clusters resulting from the activity pattern of IMG and CA thresholded at $p<.005$ will not be discussed in the paper.

Namely, we performed the correlation between BOLD signal values extracted from single-subject IMG and CA activation clusters (i.e. Model 2 and Model 3) and the LIFG activation cluster for abstract words (i.e. Model 1). A total of thirty-nine clusters (see Table 4 for cluster details) were derived from IMG and CA SPM-t maps. Only clusters exceeding an extent threshold of 10 voxels at an uncorrected p level of 0.005 are considered. Labels and percentages were obtained using the AAL toolbox (Tzourio-Mazoyer et al., 2002). Brain areas were labeled and included only when they represented at least $50 \%$ of a cluster of activation for $\mathrm{k}>50$ voxels (i.e. containing at least 26 voxels for the smallest cluster of 51 voxels), or at least $60 \%$ for a cluster extent ranging between 10-50 voxels (i.e. containing at least 6 voxels in the smallest cluster of 10 voxels). All labels were manually inspected for accuracy. Clusters are listed in decreasing order of $\mathrm{T}$ score value.

The LIFG activation cluster associated to abstract words highlighted from Model 1 and the thirtynine clusters derived from Model 2 and Model 3 were used to extract mean activation estimates from functional ROIs, defined as the entire cluster around the peak activations localized on contrast maps coding for abstract status of words (Model 1), IMG+ and IMG- (Model 2), CA+ and CA- (Model 3). The mean of activation, which represents 'typical' subject response over voxels in each region, was calculated with the SPM8 utility (EasyROI, (http://www.sbirc.ed.ac.uk/cyril/cp_download.html). 
The mean activation estimates extracted from each functional ROI for each subject were finally transformed to z-values, using the mean and standard deviation measured over BOLD response estimates across all subjects and clusters.

In order to identify the IMG and/or CA functional correlates which interact, independently or together, with the LIFG activity associated to the abstract words, pairwise BOLD correlations (functional connections) between the LIFG response in Model 1 (i.e., Y variable) and the activity extracted from the set of functional regions of interest (i.e., $\mathrm{X}$ variables) derived from Model 2 and Model 3 were carried out, generating a correlation matrix (see Table 4 for cluster correlation coefficients). Pairwise BOLD correlations of interest were assessed through Pearson Correlation analyses. In order to control for spurious associations, bootstrapping correlation confidence intervals (i.e. $n=1000$ permutations) were also calculated and only significant correlations surviving after bootstrapping method were retained. Analyses were performed with SPSS version 21 (2012).

Third, in order to trace a template activation pattern of abstractness defined respectively by IMG- or CA- and significantly associated with LIFG response to abstract words, two composite measures were created as the average of the component z-scores for the clusters corresponding respectively to IMG- and CA- activation maps shown to be significantly associated with the LIFG response to abstract words (see Table 5). Correlation analyses were used to test the relationship between the "abstractness" activation pattern subtended by imageability or context availability and LIFG activity in response to abstract words, as dichotomically opposed to concrete ones in Model 1. Time series were sampled 1,000 times through bootstrap sampling and the correlation coefficients for IMG- and CA- pattern scores and LIFG response to abstract words were computed independently.

\section{The processing of abstractness}

The logical flow and all the methodological steps performed for this section are illustrated and detailed in Supplementary Figure 4.

We aimed to pinpoint the functional network driven by the LIFG activity to abstract words for processing sensory-based perceptual (IMG-) and contextual (CA-) information. In order to reveal which LIFG-related network increases its connectivity with increases or decreases of imageability or context-availability, over and above the processing effort for words captured instead by the RT parametric modulator, we conducted psychophysiological interaction (PPI) analyses. A PPI can be identified using a linear regression model wherein a voxel's data are predicted by 1) an influencing region's data, 2) a predictor of stimulus-related signal changes, and 3) the product of the two terms 
(the interaction). If the contribution of the interaction term to the voxel measurements is significant, that implies that the contribution of the influencing region depends on the experimental context - or alternatively, that the response of the voxel to the experimental stimuli depends on the activity in the influencing region.

We used a functional ROI for the LIFG seed based on the entire cluster ( $\mathrm{k}=38$ voxels) around the peak activation $(\mathrm{x}=-53, \mathrm{y}=20, \mathrm{z}=3$ ) in the pars triangularis (BA 45) localized on the group SPM$\mathrm{t}$ map coding for abstract status of words as resulting from Model 1.

The generalized PPI toolbox (gPPI; http://www.nitrc.org/projects/gppi; McLaren et al., 2012) in SPM8 (Statistical Parametric Mapping, Wellcome Department of Cognitive Neurology, London, UK) was used for connectivity analysis. gPPI has the flexibility to accommodate multiple parametric modulators specifically the RT, the accuracy and the IMG (Model 2) or CA (Model 3) regressors in the same connectivity model.

Specifically, the gPPI toolbox was used to (1) extract the deconvolved times series from the LIFG ROI for each participant to create the physiological variables; (2) convolve each trial type and parametric modulators with the canonical HRF, creating the psychological regressor; and (3) multiply the time series from the psychological regressors with the physiological variable to create the PPI term. This interaction term identified regions that covaried in a task-dependent manner with the LIFG. For each participant, one regressor representing the deconvolved BOLD signal was included alongside each psychological and PPI term for each event type to create a gPPI model.

A whole-brain analysis (single-subject level) was then performed using the general linear model in SPM8 and two PPI contrasts were created for each model: 1) IMG+ linear increase and 2) IMG- linear increase for Model 2 and 1) CA+ linear increase and 2) CA- linear increase for Model 3 for identifying functional connectivity increases of the LIFG with other regions in the brain as 1) imageability or context availability ratings increase 2 ) imageability or context availability ratings decrease.

These individual PPI contrast images were also thresholded at voxelwise $\mathrm{p}<.005$ (cluster threshold of $\mathrm{k}=5$ ). As for the representation of abstractness, we unfolded the functional relationship between regions functionally connected with LIFG for processing sensory-based perceptual (IMG) (Model 2) or contextual (CA) (Model 3) information and LIFG neural activity necessary for differentiating 
abstract form concrete words (Model 1).

A total of fifty-four clusters (see Inline Supplementary Table 1 for cluster details) were derived from IMG and CA connectivity maps. Labels and percentages were obtained using the AAL toolbox (Tzourio-Mazoyer et al., 2002). Brain areas were labeled and included only when they represented at least $50 \%$ of a cluster of activation for $\mathrm{k}>50$ voxels (i.e. containing at least 26 voxels in the smallest cluster of 51 voxels), or at least $60 \%$ for a cluster extent ranging between 5-50 voxels (i.e. containing at least 3 voxels for the smallest cluster of 5 voxels). All labels were manually inspected for accuracy. Clusters are listed in decreasing order of $\mathrm{T}$ score values.

Mean activation estimates were derived subject-wise from the LIFG abstractlconcrete cluster highlighted from Model 1, while the 54 clusters resulting from Model 2 and Model 3 PPI analyses were used to extract connectivity strength values from clusters localized on PPI contrast maps coding for IMG+ and IMG- (Model 2) or CA+ and CA- (Model 3) using EasyROI (http://www.sbirc.ed.ac.uk/cyril/cp_download.html).

Pairwise correlations were computed between LIFG response in Model 1 (i.e. Y variable) and each region of interest (i.e., $\mathrm{X}$ variables) significantly connected with LIFG for processing imageability increases and decreases derived from Model 2 or context availability increases and decreases derived from Model 3 in order to generate a correlation matrix (see supplementary Table 1 for cluster correlation matrix).

We submitted all connectivity strength values for Pearson Correlation to calculate the correlation of interest with LIFG BOLD estimates. In addition, in order to control for spurious associations, bootstrapping correlation confidence intervals (i.e. $n=1000$ permutations) were also calculated. Only correlations deemed significant after bootstrapping were considered.

\section{Results}

\section{Behavioural Data}

In order 1) to clarify the relationship between IMG and CA for the 70 stimuli from which the stimuli were pooled and 2) to analyse the specific contribution of IMG and CA in explaining the abstractconcrete dichotomy on the $\mathrm{CNC}$ distribution, we performed both correlation and regression analyses. 
Correlations between $\mathrm{CNC}$, IMG and CA are reported in the table 3, for all the 70 stimuli and for abstract and concrete stimuli separately.

Table 3: Correlations between CNC, IMG and CA considering the entire set of stimuli, and separately for concrete and abstract words.

\begin{tabular}{|c|c|c|c|c|c|c|c|c|c|}
\hline & \multicolumn{3}{|c|}{ Experimental stimuli $(n=70)$} & \multicolumn{3}{|c|}{ Concrete stimuli $(n=35)$} & \multicolumn{3}{|c|}{ Abstract stimuli $(n=35)$} \\
\hline & CNC & IMG & $\mathrm{CA}$ & CNC & IMG & $\mathrm{CA}$ & CNC & IMG & $\mathrm{CA}$ \\
\hline CNC & & $.886^{* *}$ & $.649 * *$ & & $.417^{*}$ & .320 & & $.636^{* *}$ & $.452^{* *}$ \\
\hline IMG & $.886^{* *}$ & & $.782^{* *}$ & $.417^{*}$ & & $.603 * *$ & $.636^{* *}$ & & $.742^{* *}$ \\
\hline CA & $.649 * *$ & $.782 * *$ & & .320 & $.603 * *$ & & $.452 * *$ & $.742 * *$ & \\
\hline
\end{tabular}

CNC: concreteness; IMG: imageability; CA: Context Avalaibility. ${ }^{* *} \mathrm{p}<.001 ;{ }^{*} \mathrm{p}<.05$.

In addition, we plotted the distribution of CA and IMG for abstract and concrete words ordered on by concreteness values to better highlight the relationship between IMG and CA, see figure 1. It appears that IMG and CA do not overlap and the distance between values on each scale is shown to increase for abstract than for concrete words.

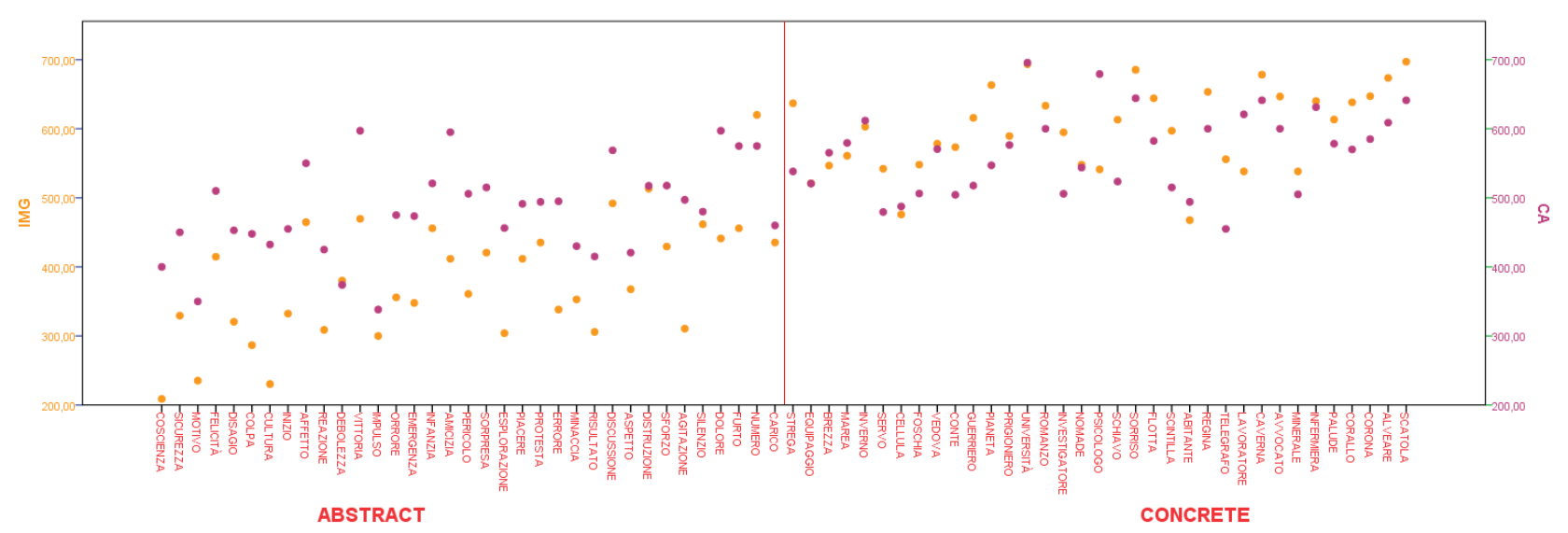

Figure 1: Distribution of the imageability and context availability values of the 70 stimuli ordered on the basis of the concreteness scale, from less concrete (abstract) to more concrete words. 
Finally, we investigated if IMG and CA explain the same part of variance of CNC. We used a residual regression technique including as independent variable IMG (i.e. the variable with the highest correlation strength) and $\mathrm{CNC}$ as dependent variable and saved unstandardized residuals. We then plotted and correlated these residuals with CA scores of the stimuli. Results show that CA is not correlated with the IMG residuals when we consider the entire sample of stimuli (p>.05), (see figure 2). When we split for abstract and concrete words, there is a significant correlation between CA and IMG residuals for both kinds of words $(r=-401, p<.05$ for abstract words and $r=-.365, p<.05$ for concrete words) (Fig 2). These results show that 1) IMG and CA may not account for the same part of variability within the CNC distribution and 2) CA captures additional variance not explained by IMG within the abstract-concrete dichotomy.

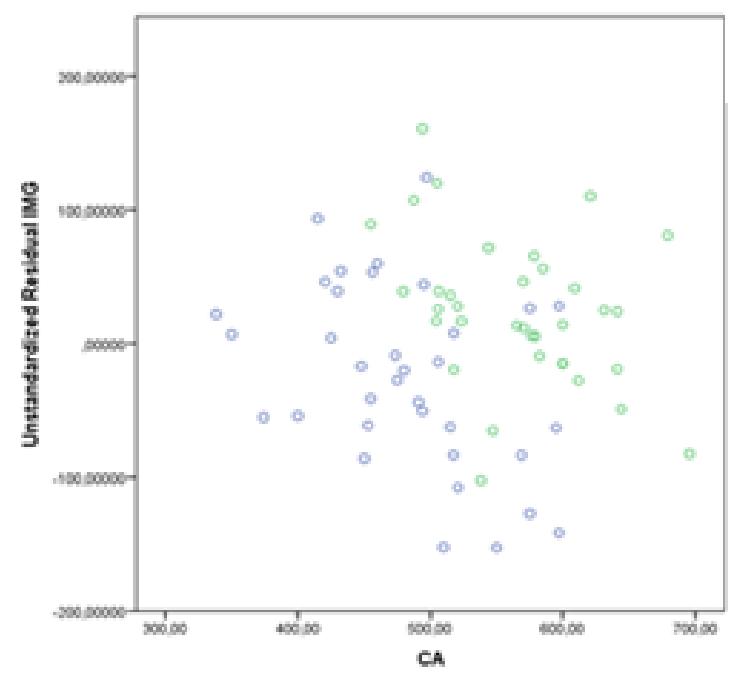

Figure 1: Relation between residuals of IMG on CNC regression analysis with CA values of stimuli. CA: Context availability; A:abstract; C:concrete.

Both reaction time and accuracy data were analyzed using a paired t-test (bootstrapped P-value where data were nonnormally distributed) in order to assess performance differences between 1) words and non-words and 2) abstract and concrete words. A difference in reaction times (words: $798 \pm 119 \mathrm{~ms}$ non-words: $929 \pm 143 \mathrm{~ms} ; \mathrm{t}=-12 ; \mathrm{p}<.001$ ) but not in accuracy (bootstrapped $\mathrm{p}=.573$ ) between words and non-words was observed. Subjects were faster (abstract: $784 \pm 117 \mathrm{~ms}$, concrete: $813 \pm 122 \mathrm{~ms}$; $\mathrm{t}=5.19 ; \mathrm{p}<.001$ ) and more accurate (abstract: $97 \%$ correct, concrete: $95 \%$ correct; bootstrapped $\mathrm{p}=.04$ ) in responding to abstract words than concrete words. A bootstrapped hierarchical regression analysis ( $n=1000$ permutations) was then carried out in order to determine which variables predict reaction times. As described above, our stimuli were balanced for several psycholinguistic variables known to 
influence task performance, with the exception of familiarity. According to the important role of emotional valence in the representation of abstract words (see Kousta et al., 2011) and the lack of matching for familiarity, we used these two variables as possible predictors. The hierarchical regression analysis included two blocks. We entered the emotional valence in Block 1, followed by familiarity in Block 2. This revealed that the emotional valence was not a significant predictor $(p=.409)$, and only familiarity significantly accounted for $38,6 \%$ of the variance $(p=.002)$.

\section{fMRI Data}

\section{The representation of abstractness}

First, the one-sample t-tests for showed that: a) for Model 1, the abstract status of words determined a significant increase in activity only in the left inferior frontal gyrus (LIFG) (pars triangularis, BA 45) (peak coordinates: $x=-53, y=20, z=3$; threshold-free cluster enhancement $[$ TFCE] $=341.16$, $\mathrm{P}=0.1, \mathrm{k}=6$ with family-wise error [FWE] correction), independent of RT; $\mathrm{b}$ ) for Model 2, the effect of "imageability" (i.e. Model 2), defined as decreasing sensory-based perceptual representations (IMG-) elicited increased activity only in the left inferior frontal gyrus (LIFG) (pars triangularis, $B A$ 45) (peak coordinates: $\mathrm{x}=-53, \mathrm{y}=18, \mathrm{z}=3$; threshold-free cluster enhancement $[\mathrm{TFCE}]=645.28$, $\mathrm{P}=0.02, \mathrm{k}=36$ with family-wise error [FWE] correction) and also for Model 3, the effect of "context availability" defined as weaker links to contextual information (CA-) triggered activation peaking in the left inferior frontal gyrus (LIFG) (pars opercularis, BA 44) (peak coordinates: $\mathrm{x}=-53, \mathrm{y}=18, \mathrm{z}$ $=3$; threshold-free cluster enhancement $[\mathrm{TFCE}]=470.08, \mathrm{P}=0.04, \mathrm{k}=20$ with family-wise error [FWE] correction), after the effects of RT were accounted for. Imcalc was then used on binarized and thresholded ( $\mathrm{p}=0.1$ FWE-corrected TFCE analysis) T-maps to determine where the statistical maps for Model 1 (i.e. abstract more than concrete), Model 2 (i.e. IMG-) and Model 3 (CA-) uniquely overlap in order to reveal the least common semantic neural denominator of abstract status, imageability and context availability. This conjunction map disclosed overlapping activity for Model 1, Model 2 and Model 3 contrast maps in LIFG (pars triangularis) (coordinates: $\mathrm{x}=-51, \mathrm{y}=22, \mathrm{z}=$ 6) (see Figure 3).

In addition, in order to translate the specific contribution of IMG and CA in explaining the abstractconcrete dichotomy on the CNC distribution form behavioral to neural terms, built an "contextavailability residual" regression model on saved unstandardized residual images generated from the 
"Imageability" model (i.e. model 2) for each subject including word trials and context availability ratings for each word as parametric modulator. On the basis of behavioral evidence showing that CA captures additional variance not explained by IMG within the abstract-concrete dichotomy, we predicted that the context availability parametric modulator should capture any residual brain activity in LIFG voxels not explained by imageability. A one-sample t-test in SPM was used on the CAcontrast images for second level group analyses using participants as a random effect and the resulting SPM-T map was thresholded at $\mathrm{p}=.05$ FWE whole-brain voxelwise corrected. Two clusters of LIFG voxels in which residual activity from Model 1 is associated to decreases in context availability were found $(x=-50, y=28, z=6 ; k=6$ voxels $-B A 45$ pars trinagularis; $x=-50, y=15, z=9 ; k=5$ voxels BA 44 pars opercularis) and are shown in supplementary Figure 3.

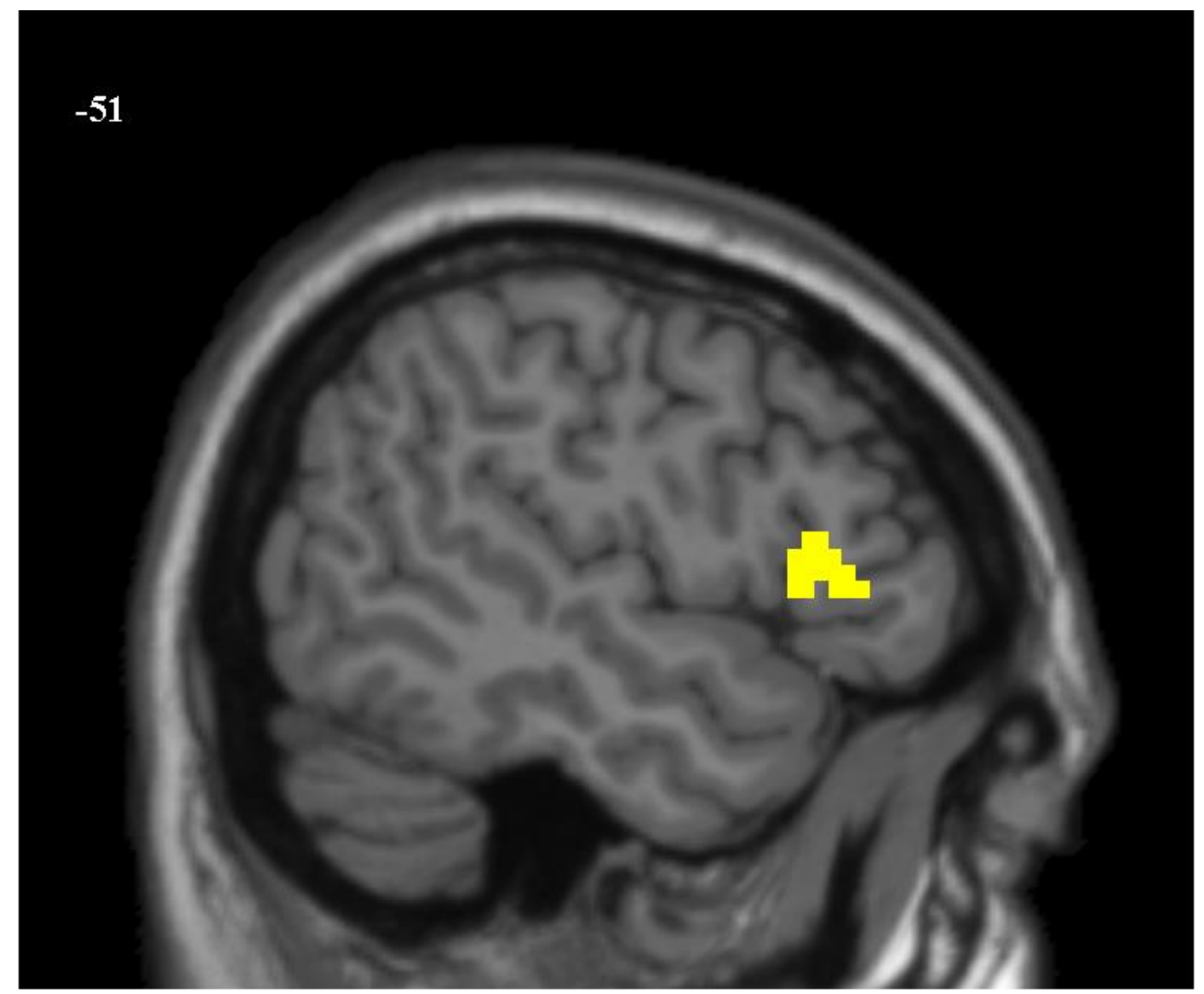

Figure 3: The conjunction between IMG- , CA- and A>C thresholded ( $p=0.1$ FWE-corrected TFCE analysis) and binarized t-maps overlaid on a sagittal slice of standard brain (i.e. the MNI-152 template) with FIVE (http://mrtools.mgh.harvard.edu/index.php?title=Main_Page). Localization of cluster local maximum $(x=-51 ; y=22 ; z=6)$ in LIFG (pars triangularis) was confirmed by Automated anatomical labeling of activations (AAL - Tzourio-Mazoyer et al., 2002) in FIVE (http://mrtools.mgh.harvard.edu/index.php?title=Main_Page). 
Second, we elucidated the functional association between imageability (Model 2) and context availability (Model 3) regions with LIFG activation cluster for abstract words, highlighted in Model 1. For Model 2, only activation in a subset of functional ROIs sensitive to decreases in imageability (IMG-), mainly localized in the left hemisphere, including the fusiformlingual gyrus ( $\mathrm{r}=.449,95 \%$ $\mathrm{CI}=0.026$ to 0.710$)$, the mid-occipital gyrus $(\mathrm{r}=.570,95 \% \mathrm{CI}=0.324$ to 0.805$)$ and SMA $(\mathrm{r}=.611,95 \%$ $\mathrm{CI}=0.349$ to 0.772 ), added to the right mid-superior occipital gyrus ( $\mathrm{r}=.496,95 \% \mathrm{CI}=0.263$ to 0.788 ), was significantly positively correlated with the LIFG response to abstract words (see Table 4).

For Model 3, only brain activity in a few clusters responding to decreasing context availability (CA) and located exclusively in the right hemisphere, involving the lingual/fusiform gyrus ( $\mathrm{r}=.429,95 \%$ $\mathrm{CI}=0.124$ to 0.712 ), the rolandic operculumlinsula ( $\mathrm{r}=.396,95 \% \mathrm{CI}=0.073$ to 0.633$)$ and the $\mathrm{IFG}$ (pars opercularis Itriangularis) ( $\mathrm{r}=.662,95 \% \mathrm{CI}=0.185$ to 0.861$)$ was significantly positively correlated with the LIFG response to abstract words (see Table 4).

\begin{tabular}{|c|c|c|c|c|c|}
\hline Condition & $\begin{array}{l}\text { cluster } \\
\text { extent }\end{array}$ & coordinates & $\mathrm{t}$ value & Region & correlation with LIFG $(A>C)$ \\
\hline IMG + & 16 & 453327 & 3,84 & R MIDDLE FRONTAL GYRUS & Ns \\
\hline IMG - & 115 & $-60-430$ & 5,25 & L MIDDLE TEMPORAL GYRUS & Ns \\
\hline IMG - & 24 & $10-850$ & 4,94 & R CALCARINE GYRUS & Ns \\
\hline IMG - & 28 & -4033 & 4,70 & L INSULA & Ns \\
\hline IMG - & 23 & $-28-68-6$ & 4,66 & L FUSIFORM GYRUS & $r=.449 ; p=.02 *(C l: 0.026-0.710)$ \\
\hline IMG - & 38 & $-20-906$ & 4,50 & L MIDDLE OCCIPITAL GYRUS & Ns \\
\hline IMG - & 92 & $30-8815$ & 4,17 & R MIDDLE OCCIPITAL GYRUS & $r=.496 ; p=.01 * *(C l: 0.263-0.788)$ \\
\hline IMG - & 23 & -35836 & 3,83 & L PRECENTRAL GYRUS & Ns \\
\hline IMG - & 21 & $-30-7521$ & 3,76 & L MIDDLE OCCIPITAL GYRUS & $r=.570 ; p=.002 * *(C l: 0.324-0.805)$ \\
\hline IMG - & 32 & -31060 & 3,73 & LSMA & $r=.611 ; p=.001 * *(C l: 0.349-0.772)$ \\
\hline IMG - & 10 & 25689 & 3,61 & R CALCARINE GYRUS & Ns \\
\hline $\mathrm{CA}+$ & 29 & $48-5536$ & 4,54 & R ANGULAR GYRUS & Ns \\
\hline $\mathrm{CA}+$ & 48 & $0-6533$ & 3,55 & L PRECUNEUS & Ns \\
\hline $\mathrm{CA}+$ & 20 & -252351 & 3,44 & L MIDDLE FRONTAL GYRUS & Ns \\
\hline $\mathrm{CA}+$ & 10 & $8-3833$ & 3,39 & R MIDDLE CINGULATE CORTEX & Ns \\
\hline
\end{tabular}




\begin{tabular}{|c|c|c|c|c|c|}
\hline CA+ & 12 & $63-25-6$ & 3,39 & R MIDDLE TEMPORAL GYRUS & Ns \\
\hline $\mathrm{CA}+$ & 10 & $-40-5833$ & 3,27 & L ANGULAR GYRUS & Ns \\
\hline CA - & 258 & $-5-2551$ & 5,11 & L PARACENTRAL LOBULE & Ns \\
\hline CA - & 39 & -4050 & 4,97 & L INSULA & Ns \\
\hline CA - & 25 & $23-75-3$ & 4,73 & R LINGUAL GYRUS & $r=.429 ; p=0,029 *(C l: 0.124-0.712)$ \\
\hline CA - & 84 & 581315 & 4,67 & R INFERIOR FRONTAL GYRUS (pars opercularis) & Ns \\
\hline CA - & 139 & $8-3354$ & 4,51 & R PARACENTRAL LOBULE & Ns \\
\hline CA - & 40 & $-60-403$ & 4,48 & L MIDDLE TEMPORAL GYRUS & Ns \\
\hline CA - & 33 & 354830 & 4,40 & R MIDDLE FRONTAL GYRUS & Ns \\
\hline CA - & 20 & $18-2869$ & 4,23 & R PRECENTRAL GYRUS & Ns \\
\hline CA - & 54 & $38-70-9$ & 4,22 & R INFERIOR OCCIPITAL GYRUS & Ns \\
\hline CA - & 18 & $-23-2566$ & 4,19 & L PRECENTRAL GYRUS & Ns \\
\hline CA - & 50 & $-28-68-6$ & 4,12 & L FUSIFORM GYRUS & Ns \\
\hline CA - & 10 & $15-5066$ & 4,05 & R SUPERIOR PARIETAL LOBULE & Ns \\
\hline CA - & 41 & $28-53-15$ & 4,04 & R FUSIFORM GYRUS & Ns \\
\hline CA - & 17 & $-48-1815$ & 3,95 & L ROLANDIC OPERCULUM & Ns \\
\hline CA - & 15 & $43-1515$ & 3,92 & R ROLANDIC OPERCULUM & $r=.396 ; p=0,045^{*}(\mathrm{Cl}: 0,073-0.633)$ \\
\hline CA - & 24 & $40-3354$ & 3,86 & R POSTCENTRAL GYRUS & Ns \\
\hline CA - & 41 & $-28-8027$ & 3,69 & L MIDDLE OCCIPITAL GYRUS & Ns \\
\hline CA - & 15 & $-30-560$ & 3,58 & L PRECENTRAL GYRUS & Ns \\
\hline CA - & 13 & $38-357$ & 3,51 & R MIDDLE FRONTAL GYRUS & Ns \\
\hline CA - & 14 & $-23-4366$ & 3,40 & L POSTCENTRAL GYRUS & Ns \\
\hline CA - & 19 & 50183 & 3,34 & R INFEROR FRONTAL GYRUS (p. opercularis) & $r=.662 ; p=0,000 * *(C l: 0.185-0.861)$ \\
\hline CA - & 11 & $-55-3330$ & 3,30 & L SUPRAMARGINAL GYRUS & Ns \\
\hline
\end{tabular}

Table 4. Location and MNI coordinates of peaks of significant activation for IMG+ e IMG- (in light grey) and for CA+ e CA(dark grey). Cluster extent is indicated by $\mathrm{k}=$ number of voxels in the particular cluster. Labels and percentages were obtained using the AAL toolbox (Tzourio-Mazoyeret al., 2002). p values of the Pearson $r$ correlation coefficients and bootstrap ( $n=1000$ samples) 95\% confidence intervals $(\mathrm{Cl})$ are reported for pairwise BOLD functional correlations between each cluster of activation found for Model 2 and Model 3 and LIFG BOLD response resulting from Model 1. Significant correlations are reported in bold.

$* *=$ Correlation is significant at the .01 level (2-tailed).

$*=$ Correlation is significant at the .05 level (2-tailed).

Ns=not significant

The positive correlation between both the imageability ( $\mathrm{r}=.689, \mathrm{p}=.01,95 \% \mathrm{CI}=.548$ to .826$)$ and context availability $(\mathrm{r}=.701, \mathrm{p}=.01,95 \% \mathrm{CI}=.369$ to .871$)$ composite measures with the LIFG response to abstract words was significant. 
We further confirmed the functional relationship between the IMG-and CA- abstractness fingerprints and the LIFG dichotomic response to abstract words using a linear multiple stepwise regression analysis. The IMG- and CA- composite measures were used as regressors to predict BOLD signal estimates in LIFG for abstract status of words. The stepwise methodology used $p=0.05$ to enter IMGand CA- scores into each step and $\mathrm{p}=0.1$ to remove. The final model incorporated both the IMGand CA- predictors $\left(\mathrm{R}^{2}=.58\right.$, adjusted $\left.\mathrm{R}^{2}=.54, F(2,23)=15.8, p<.001\right)$ with the partial correlations for each of these variables explaining significant portions of the variance (see Table 5). Inspection of the beta weights revealed that LIFG response to abstract words increased as overall activation increases in the abstractness template pattern defined by both IMG- and CA- .

Table 5

\begin{tabular}{lcccccc} 
Predictor (composite measure) & Beta (raw) & Beta (stand) & t value & Semipartial $R^{2}$ & $p$ value \\
& & & & & & \\
\hline IMG- & 1.27 & .40 & 2.20 & .30 & .038 \\
CA- & .49 & .43 & 2.39 & .32 & .025 \\
\hline
\end{tabular}

Table 5: Results of a stepwise regression analyses

\section{The processing of abstractness}

First, we have portrayed the abstractness template pattern of activation traced by both sensory-based perceptual (IMG-) and contextual (CA-) information and established that both have a functional relationship with the specific neural activity elicited in LIFG necessary for differentiating abstract from concrete words. Our second aim was to pinpoint the functional network driven by the LIFG activity to abstract words for processing sensory-based perceptual (IMG-) and contextual (CA-) information. In order to reveal which LIFG-related network increases its connectivity with increases or decreases of imageability or context-availability, over and above the processing effort for words captured instead by the RT parametric modulator, we conducted psychophysiological interaction (PPI) analyses.

The PPI results showed that the only significant correlation was for two IMG- clusters derived from PPI analysis with Model 2. The first is located in the left precentral $\backslash$ mid-frontal gyrus $(x=-42, y=2$, $\mathrm{z}=48)(\mathrm{r}=-.456,95 \% \mathrm{CI}=-.699$ to $-.017, \mathrm{p}=.02)$ and the second falls within an area encompassing the 
left middle temporal and angular gyrus $(\mathrm{x}=-50, \mathrm{y}=-52, \mathrm{z}=15)(\mathrm{r}=-.476,95 \% \mathrm{CI}=-.704$ to -.215 , $\mathrm{p}=.014)$.

A stepwise multiple regression including LIFG-connectivity strength values relative to processing imageability decreases for both clusters was conducted to determine if LIFG-connectivity with either one region independently predicted changes in LIFG BOLD estimates.

The stepwise multiple regression model incorporated only the left mid-superior temporallangular gyrus cluster $\left(\mathrm{R}^{2}=.23\right.$, adjusted $\left.\mathrm{R}^{2}=.19, \mathrm{~F}(1,24)=7.02, \mathrm{p}=.014\right)$. The left precentral $\backslash$ mid-frontal gyrus cluster failed to explain a significant amount of additional variance. The negative beta weight (raw $=-.97$, standardized $=-.48$ ) indicates that the significant decreased LIFG-connectivity with the left middle temporallangular gyrus cluster for processing increases in imageability is significantly mirrored by the significant increases in activation in the LIFG necessary to dichotomize between abstract and concrete words. 


\section{Discussion}

In this study, we aimed at unravelling 1) the functional correlations of the intrinsic and extrinsic properties of abstract and concrete representations, namely imageability (IMG) and context availability (CA) with brain activation differentiating abstract with respect to concrete representations; 2) and if the left IFG may act as a functional convergence zone between IMG and $\mathrm{CA}$, reconciling the abstract/concrete neural dichotomy.

A lexical decision task has been employed, revealing that abstract words were processed faster than concrete words. This result has been already reported by previous studies in which abstract and concrete word were matched for several psycholinguistic variables (Kousta et al., 2011), generally not considered in most of the fMRI studies. According to Kousta et al., emotional valence plays a crucial role in the processing and representation of abstract concepts, predicting the advantage for abstract words in their experiment, where abstract words were more emotionally valenced than concrete words. In our study, emotional valence, as well as several other psycholinguistic variables, with the exception of familiarity (with abstract words more familiar than concrete words), were balanced between abstract and concrete words. Reaction times are predicted only by familiarity. However, in all fMRI analyses, we used RTs as predictor in order to control for task demands.

Our results highlight the prominent role of the left IFG in differentiating abstract from concrete processing, in line with several studies on this issue (Perani et al., 1999; Binder et al., 2005; Noppeney \& Price, 2004; Fiebach \& Friederici, 2003; Friederici et al., 2000; Jessen et al., 2000; Papagno et al., 2009; Shallice \& Cooper, 2013). Different interpretations have been however reported to account for the role of the left IFG in abstract processing. Some researchers interpreted the IFG involvement linked to a verbal semantic network supporting abstract representations (Wang et al., 2010; see also Goldberg et al., 2007), as predicted by the Dual-coding Theory (Paivio, 1971, 1985). Other researchers have attributed the greater activation to the fact that for abstract words appear in a wider range of contexts and consequently are likely to have more complex and variable meanings, requiring consequently greater executive regulation (Hoffman et al., 2015; Fiebach and Friederici, 2004; Noppeney and Price, 2004), in accordance with the Context Availability Theory (Schwanenflugel and Shoben, 1983).

Making reference to these distinctions, and in order to define imaegability and context availability as intrinsic and extrinsic properties involved in representing abstract concepts meanings, we operationalized these two types of information in terms of low imageability (i.e., linked to verbal content) and in terms of low context availability (Della Rosa et al., 2011). 
A conjunction analysis shows a common activation for words with low IMG or low CA only in the LIFG, in the same area reported for abstract words; a result compatible with both interpretations, as both low IMG and low CA are implicated in abstract knowledge processing. However, functional activation maps for low level of CA and low level of IMG reveal also partially different circuits with only some of these regions functionally correlated with left IFG activation accounting for abstract words processing. In particular, among the regions associated to low IMG, those functionally correlated with the left IFG were mainly left lateralized, including bilateral middle occipital gyri, the left fusiform gyrus and the left SMA, while those associated with low CA had a right lateralization and included lingual gyrus, the rolandic operculum and the IFG.

The different lateralization for low IMG and low CA may be linked to previous proposals that have argued for a different role of the left and the right hemispheres in language comprehension. While the left hemisphere selects closely related meanings and a single interpretation, the right hemisphere actives a much broader range of related meanings, including those that are less related or contextually inconsistent, metaphoric interpretations, and multiple meanings of ambiguous words (e.g., Anaki, 1998; Beeman, 1998; Burgess \& Simpson, 1988; Faust \& Chiarello, 1998; Faust \& Gernsbacher, 1996; Jung-Beeman et al., 2004; Klepousniotou \& Baum, 2005; Faust \& Kenneth , 2014).

According to this view, the LIFG may be equally sensitive to both types of information, but in a different way and through different hemispheres. On the one and "left" hand it might intrinsically facilitate recognition of higher-order semantic relations supporting semantic integration by allowing the brain to refine, elaborate and integrate abstract meanings. On the other and "right" hand it may extrinsically focus semantic activation on features related to the dominant or contextually relevant meaning while inhibiting features related to the subordinate or contextually irrelevant meanings, while selecting one abstract concept with respect to another.

As abstract words can occur in many different contexts with associated variations in meaning, according to other authors, the right hemisphere could be also be expected to manage stronger demands on executive semantic control processes (Noonan et al., 2013). Moreover, both functional neuroimaging and patient studies reported that regions on the right hemisphere contribute to tasks with high semantic control demands (Noonan et al., 2013; Thompson et al., 2016). Accordingly, functional connectivity between the left and right IFG has been reported when semantic tasks requiring more control were contrasted with more automatic semantic retrieval, suggesting that the right IFG is part of the semantic control network (Hallam et al., 2016; Noonan et al., 2013). 
Our results, according to previous studies (Perani et al., 1999; Kiehl et al., 1999), report an involvement of the right IFG in abstract processing, suggesting an involvement of this region associated with low CA, namely with a greater difficulty to think of a context. Our results do not fit with an interpretation in term of executive requirements due to greater cognitive load, as they were independent of RTs.

We also examined the pattern of functional connectivity for LIFG specifically involved in both low IMG and low CA in order to solve the lexical decision task using psychophysical interaction models. On the basis of this PPI analysis, connectivity strength values extracted from each region recruited by low IMG or low CA and connected with the LIFG were used to predict the greater activation of the IFG for abstract concepts. Only the left posterior middle temporal gyrus (pMTG)/angular gyrus, specifically associated to low IMG, was a significant predictor of LIFG activity differentiating abstract from concrete words.

Both the pMTG and the angular gyrus have been classically involved in language comprehension and semantic processing (Benson, 1979; Martin, 2007; Binder et al., 2009; Schwartz et al., 2011; Bonner et al., 2013). While a greater activation of the pMTG has been reported for abstract than concrete concepts (Grossman et al., 2002; Noppeney and Price, 2004; Pexman et al., 2007; Sabsevitz et al., 2005; Wang et al., 2010); the angular gyrus has been reported to be more active in response to concrete over abstract word processing (Binder et al., 2009; Wang et al., 2010). More recently, however, a functional connectivity between the left IFG and the left temporo-parietal junction (TPJ), including the superior and middle temporal gyri and the supramarginal and angular gyri, has been reported during abstract concept processing (Skipper-Kallal et al., 2015), suggesting a key role of the left TPJ in comprehension of abstract concepts.

The left pMTG and angular gyrus have been also recruited in cases of high-control semantic conditions (Noonan et al, 2013). A greater activation of pMTG, generally reported together with the left IFG during high-control semantic conditions, may reflect an additional activation of conceptual knowledge on demanding trials (Badre et al., 2005), or may act as a part of the semantic control network (Noonan et al., 2013; Davey et al., 2015). Davey et al. (2016) in their fMRI study on concrete concepts suggested that the pMTG, together with the more anterior/ventral portion of the IFG, acts as a functional nexus facilitating the integration of information from regions of the default mode network, associated with more automatic aspects of semantic cognition, and those in the multipledemand network, generally associated with executive-demanding tasks. 
According to recent evidence, different portions of the angular gyrus contribute to different functions. While the more dorsal portions are involved in the semantic control, the middle and ventral regions play a key role in semantic processing irrespective of control demands (Noonan et al., 2013; Davey et al., 2015).

Our results do not support an involvement of pMTG/angular gyrus in the semantic control network, nor a role of functional nexus, at least for abstract concept processing.

Our analysis in fact identified an involvement of the more ventral regions of angular gyrus in line with a role in semantic processing (Noonan et al., 2013). In addition, the greater activation of the left IFG for abstract concepts was predicted only by the left pMTG/ angular gyrus associated to low IMG, and not for low $\mathrm{CA}$, and over and above the processing effort for words captured by the RT parametric modulator. In addition, the pMTG activation was not reported in the conjunction analysis considering abstract words, low IMG and low CA conditions.

The semantic processing invoked in completing the lexical decision task taps words' intrinsic properties, involving a left-lateralized network focusing and selecting the semantic activation related to the dominant meaning of the words. This semantic activation is conducive to most language tasks involving single words (Jung-Beeman, 2005). The lexical decision task does not require a coarse semantic coding supported by the right hemisphere (Jung-Beeman, 2005). However, participants construct different semantic representations as a function of task demands, so it is certainly possible that extrinsic semantic dimensions could be important in different tasks or with different item sets (for example in the summation tasks and task-irrelevant information, see Thompson et al., 2016). Thus, in the case of lexical decision on abstract words the IFG engagement is driven to a higher degree by the network supporting low IMG with respect to regions related to low CA, suggesting that the LIFG-pMTG coupling in abstract concept processing is the result of activation of the language based or semantic (verbal) system (Wang et al., 2010).

In conclusion we showed different functional associations and connectivity strength between areas in the activity pattern for IMG or CA with a shared LIFG activation between abstract, less imageable and less contextually available words. Our hypothesis is that the activation in LIFG is the global result of the selected variables, which are all involved in the representation of abstractness. These two representations may engage the LIFG differently, and that this conjoint activity in LIFG is just the shared tip of an iceberg, which appears in many studies but that can be subtended by at least a partially different activity pattern depending on the characteristics of the information (intrinsic or extrinsic) necessary to represent and process word meaning. 
Our results support the idea that the LIFG can be identified as the neural crossroads for representing and processing abstract knowledge in order to differentiate between abstract and concrete concepts. Abstract conceptual processing requires an interplay of multiple brain regions necessary for both the semantic representation of abstract knowledge and for higher taxing on executive control processing exerted by the stimuli. The results support the role of the IFG as an effector/mediator of both intrinsic (semantic content) and extrinsic (associated contexts/semantic control) proprieties of abstract concepts, reconciling long-standing disputes about the nature of its role. Thus, we propose a further account of the role of the left IFG in the semantic processing of abstract concepts.

In our view, abstract words require that elements defining both the intrinsic and extrinsic properties of word meaning need to overlap to a sufficient degree in order to be unified in consistent to-becombined representations for abstract meanings (Shallice \& Cooper 2013), where imageability can functionally serve for semantic integration and context availability for semantic selection.

Its role is in line with Hagoort's proposal about the unification role of this area (BA 45 and BA 47), in integrating or "unifying" semantic knowledge of a word with prior context (Hagoort, 2005; Hagoort et al., 2016), thanks to the interaction with the other regions, which are part of the networks accounting for both intrinsic and extrinsic properties. Our view is also in accordance with the idea that abstract representations in the brain are the result of a process of hierarchical conjunctive coding, and "it is their combinatorial nature that is important rather than their abstractness per se" (Binder, 2016).

\section{Acknowledgements}

This study was made possible through the support of a grant from European Union (FP6-2004NESTPATH, grant 028714 to S.C. and G.V.) and from ESRC (grant RES-062-23-2012 to G.V.).

\section{References}

Anaki, D., Faust, M., \& Kravetz, S. (1998). Cerebral hemispheric asymmetries in processing lexical metaphors. Neuropsychologia, 36(4), 353-362.

Badre, D., Poldrack, R. A., Paré-Blagoev, E. J., Insler, R. Z., \& Wagner, A. D. (2005). Dissociable controlled retrieval and generalized selection mechanisms in ventrolateral prefrontal cortex.

Neuron, 47(6), 907-918. 
Beeman, M. (1998). Coarse semantic coding and discourse comprehension. Right hemisphere language comprehension: perspectives from cognitive neuroscience, ed. by M. Beeman and C. Chiarrello, 51-78.

Benson, D. F. (1979). Aphasia, alexia, and agraphia (Vol. 1). Churchill Livingstone.

Binder, J. R., Conant, L. L., Humphries, C. J., Fernandino, L., Simons, S. B., Aguilar, M., \& Desai, R. H. (2016). Toward a brain-based componential semantic representation. Cognitive neuropsychology, 33(3-4), 130-174.

Binder, J. R., Desai, R. H., Graves, W. W., \& Conant, L. L. (2009). Where is the semantic system? A critical review and meta-analysis of 120 functional neuroimaging studies. Cerebral Cortex, 19(12), 2767-2796.

Binder, J. R., Desai, R. H., Graves, W. W., \& Conant, L. L. (2009). Where is the semantic system? A critical review and meta-analysis of 120 functional neuroimaging studies. Cerebral Cortex, 19(12), 2767-2796.

Binder, J. R., Westbury, C. F., McKiernan, K. A., Possing, E. T., \& Medler, D. A. (2005). Distinct brain systems for processing concrete and abstract concepts. Journal of cognitive neuroscience, 17(6), 905-917.

Bonner, M. F., Peelle, J. E., Cook, P. A., \& Grossman, M. (2013). Heteromodal conceptual processing in the angular gyrus. Neuroimage, 71, 175-186.

Burgess, C., \& Simpson, G. B. (1988). Cerebral hemispheric mechanisms in the retrieval of ambiguous word meanings. Brain and language, 33(1), 86-103.

Cortese, M. J., \& Khanna, M. M. (2007). Age of acquisition predicts naming and lexical-decision performance above and beyond 22 other predictor variables: An analysis of 2,342 words. The Quarterly Journal of Experimental Psychology, 60(8), 1072-1082.

Dale, A. M. (1999). Optimal experimental design for event-related fMRI. Human brain mapping, 8(2-3), 109-114.

Davey, J., Cornelissen, P. L., Thompson, H. E., Sonkusare, S., Hallam, G., Smallwood, J., \& Jefferies, E. (2015). Automatic and controlled semantic retrieval: TMS reveals distinct contributions of posterior middle temporal gyrus and angular gyrus. Journal of neuroscience, 35(46), 1523015239.

Davey, J., Thompson, H. E., Hallam, G., Karapanagiotidis, T., Murphy, C., De Caso, I., KriegerRedwood, K,. Bernhardt, C,.B., Smallwood, J., \& Jefferies, E. (2016). Exploring the role of the posterior middle temporal gyrus in semantic cognition: Integration of anterior temporal lobe with executive processes. NeuroImage, 137, 165-177.

Della Rosa, P. A., Catricalà, E., Vigliocco, G., \& Cappa, S. F. (2010). Beyond the abstractconcrete dichotomy: Mode of acquisition, concreteness, imageability, familiarity, age of acquisition, context availability, and abstractness norms for a set of 417 Italian words. Behavior research methods, 42(4), 1042-1048.

Faust, M. E., \& Gernsbacher, M. A. (1996). Cerebral mechanisms for suppression of inappropriate information during sentence comprehension. Brain and language, 53(2), 234-259. 
Faust, M., \& Chiarello, C. (1998). Sentence context and lexical ambiguity resolution by the two hemispheres. Neuropsychologia, 36(9), 827-835.

Faust, M., \& Kenett, Y. N. (2014). Rigidity, chaos and integration: hemispheric interaction and individual differences in metaphor comprehension. Frontiers in human neuroscience, 8.

Fiebach, C. J., \& Friederici, A. D. (2004). Processing concrete words: fMRI evidence against a specific right-hemisphere involvement. Neuropsychologia, 42(1), 62-70.

Fiebach, C. J., Friederici, A. D., Müller, K., \& Von Cramon, D. Y. (2002). fMRI evidence for dual routes to the mental lexicon in visual word recognition. Journal of cognitive neuroscience, 14(1), $11-23$.

Friederici, A. D., Opitz, B., \& Von Cramon, D. Y. (2000). Segregating semantic and syntactic aspects of processing in the human brain: an fMRI investigation of different word types. Cerebral cortex, 10(7), 698-705.

Goldberg, R. F., Perfetti, C. A., Fiez, J. A., \& Schneider, W. (2007). Selective retrieval of abstract semantic knowledge in left prefrontal cortex. Journal of Neuroscience, 27(14), 3790-3798.

Grossman, M., Koenig, P., DeVita, C., Glosser, G., Alsop, D., Detre, J., \& Gee, J. (2002). The neural basis for category-specific knowledge: an fMRI study. Neuroimage, 15(4), 936-948.

Hagoort, P. (2005). On Broca, brain, and binding: a new framework. Trends in cognitive sciences, 9(9), 416-423.

Hagoort, P. (2016). MUC (Memory, Unification, Control): A model on the neurobiology of language beyond single word processing. In Neurobiology of language (pp. 339-347). Elsever.

Hallam, G. P., Whitney, C., Hymers, M., Gouws, A. D., \& Jefferies, E. (2016). Charting the effects of TMS with fMRI: Modulation of cortical recruitment within the distributed network supporting semantic control. Neuropsychologia, 93, 40-52.

Hoffman, P., Binney, R. J., \& Ralph, M. A. L. (2015). Differing contributions of inferior prefrontal and anterior temporal cortex to concrete and abstract conceptual knowledge. Cortex, 63, 250-266.

Jessen, F., Heun, R., Erb, M., Granath, D. O., Klose, U., Papassotiropoulos, A., \& Grodd, W. (2000). The concreteness effect: Evidence for dual coding and context availability. Brain and language, 74(1), 103-112.

Jung-Beeman, M. (2005). Bilateral brain processes for comprehending natural language. Trends in cognitive sciences, 9(11), 512-518.

Jung-Beeman, M., Bowden, E. M., Haberman, J., Frymiare, J. L., Arambel-Liu, S., Greenblatt, Reber, P., J.. \& Kounios, J. (2004). Neural activity when people solve verbal problems with insight. PLoS biology, 2(4), e97.

Kiehl, K. A., Liddle, P. F., Smith, A. M., Mendrek, A., Forster, B. B., \& Hare, R. D. (1999). Neural pathways involved in the processing of concrete and abstract words. Human brain mapping, 7(4), 225-233. 
Klepousniotou, E., \& Baum, S. R. (2005). Processing homonymy and polysemy: Effects of sentential context and time-course following unilateral brain damage. Brain and Language, 95(3), 365-382.

Kousta, S. T., Vigliocco, G., Vinson, D. P., Andrews, M., \& Del Campo, E. (2011). The representation of abstract words: why emotion matters. Journal of Experimental Psychology: General, 140(1), 14.

Liakakis, G., Nickel, J., \& Seitz, R. J. (2011). Diversity of the inferior frontal gyrus - a metaanalysis of neuroimaging studies. Behavioural brain research, 225(1), 341-347.

Martin, A. (2007). The representation of object concepts in the brain. Annu. Rev. Psychol., 58, 2545 .

McLaren, D. G., Ries, M. L., Xu, G., \& Johnson, S. C. (2012). A generalized form of contextdependent psychophysiological interactions (gPPI): a comparison to standard approaches. Neuroimage, 61(4), 1277-1286.

Noonan, K. A., Jefferies, E., Visser, M., \& Ralph, M. A. L. (2013). Going beyond inferior prefrontal involvement in semantic control: evidence for the additional contribution of dorsal angular gyrus and posterior middle temporal cortex. Journal of cognitive neuroscience, 25(11), 1824-1850.

Noppeney, U., \& Price, C. J. (2004). Retrieval of abstract semantics. Neuroimage, 22(1), 164-170.

Paivio, A. (1986). Mental representations: A dual coding approach. Oxford, England: Oxford University Press.

Paivio, A. (1971). Imagery and language. Imagery: Current cognitive approaches, 7-32.

Paivio, A. (1985). Cognitive and motivational functions of imagery in human performance. Canadian journal of applied sport sciences. Journal canadien des sciences appliquées au sport, $10(4), 22 \mathrm{~S}-28 \mathrm{~S}$.

Paivio, A. (1991). Dual coding theory: Retrospect and current status. Canadian journal of psychology, 45(3), 255-287.

Paivio, A. (2010). Dual coding theory and the mental lexicon. The Mental Lexicon, 5(2), 205-230.

Paivio, A., Yuille, J. C., \& Madigan, S. A. (1968). Concreteness, imagery, and meaningfulness values for 925 nouns. Journal of experimental psychology, 76(1p2), 1.

Papagno, C., Fogliata, A., Catricalà, E., \& Miniussi, C. (2009). The lexical processing of abstract and concrete nouns. Brain research, 1263, 78-86.

Perani, D., Cappa, S. F., Schnur, T., Tettamanti, M., Collina, S., Rosa, M. M., \& Fazio1, F. (1999). The neural correlates of verb and noun processing: A PET study. Brain, 122(12), 2337-2344.

Pexman, P. M., Hargreaves, I. S., Edwards, J. D., Henry, L. C., \& Goodyear, B. G. (2007). Neural correlates of concreteness in semantic categorization. Journal of Cognitive Neuroscience, 19(8), 1407-1419.

Random Word Generator. https://www.gammadyne.com/rndword.htm 
Sabsevitz, D. S., Medler, D. A., Seidenberg, M., \& Binder, J. R. (2005). Modulation of the semantic system by word imageability. Neuroimage, 27(1), 188-200.

Schwanenflugel, P. J., \& Shoben, E. J. (1983). Differential context effects in the comprehension of abstract and concrete verbal materials. Journal of Experimental Psychology: Learning, Memory, and Cognition, 9(1), 82.

Schwanenflugel, P. J., \& Shoben, E. J. (1983). Differential context effects in the comprehension of abstract and concrete verbal materials. Journal of Experimental Psychology: Learning, Memory, and Cognition, 9(1), 82.

Schwanenflugel, P. J., Harnishfeger, K. K., \& Stowe, R. W. (1988). Context availability and lexical decisions for abstract and concrete words. Journal of Memory and Language, 27(5), 499-520.

Schwartz, M. F., Kimberg, D. Y., Walker, G. M., Brecher, A., Faseyitan, O. K., Dell, G. S., ... \& Coslett, H. B. (2011). Neuroanatomical dissociation for taxonomic and thematic knowledge in the human brain. Proceedings of the National Academy of Sciences, 108(20), 8520-8524.

Shallice, T., \& Cooper, R. P. (2013). Is there a semantic system for abstract words?. Frontiers in human neuroscience, 7 .

Skipper-Kallal, L. M., Mirman, D., \& Olson, I. R. (2015). Converging evidence from fMRI and aphasia that the left temporoparietal cortex has an essential role in representing abstract semantic knowledge. Cortex, 69, 104-120.

SPM8. http://www.fil.ion.ucl.ac.uk/spm

Spss, I. (2012). IBM SPSS statistics version 21. Boston, Mass: International Business Machines Corp, 126.

Thompson, H. E., Henshall, L., \& Jefferies, E. (2016). The role of the right hemisphere in semantic control: A case-series comparison of right and left hemisphere stroke. Neuropsychologia, 85, 44-61.

Vigliocco, G., Kousta, S. T., Della Rosa, P. A., Vinson, D. P., Tettamanti, M., Devlin, J. T., \& Cappa, S. F. (2013). The neural representation of abstract words: the role of emotion. Cerebral Cortex, 24(7), 1767-1777.

Vigliocco, G., Meteyard, L., Andrews, M., \& Kousta, S. (2009). Toward a theory of semantic representation. Language and Cognition, 1(2), 219-247.

Wang, J., Conder, J. A., Blitzer, D. N., \& Shinkareva, S. V. (2010). Neural representation of abstract and concrete concepts: A meta-analysis of neuroimaging studies. Human brain mapping, 31(10), 1459-1468.

Weiskopf, N., Hutton, C., Josephs, O., \& Deichmann, R. (2006). Optimal EPI parameters for reduction of susceptibility-induced BOLD sensitivity losses: a whole-brain analysis at $3 \mathrm{~T}$ and 1.5 T. Neuroimage, 33(2), 493-504. 
Inline Supplementary Table 1

\begin{tabular}{ccccclc}
\hline Condition & $\begin{array}{c}\text { Cluster } \\
\text { label }\end{array}$ & $\begin{array}{c}\text { cluster } \\
\text { extent }\end{array}$ & coordinates & t value & Region & correlation with LIFG (A>C) \\
\hline IMG + & CL1 & 14 & -17106 & 3,72 & L PUTAMEN & $n s$ \\
IMG + & CL2 & 7 & 255227 & 3,50 & R MIDDLE FRONTAL GYRUS & $n$ ns \\
IMG- & CL1 & 81 & $-50-20-9$ & 5,91 & L MIDDLE TEMPORAL GYRUS & ns \\
IMG- & CL4 & 40 & $20-6212$ & 4,73 & R CALCARINE GYRUS & ns
\end{tabular}




\begin{tabular}{|c|c|c|c|c|c|c|}
\hline IMG- & CL6 & 6 & -45248 & 4,25 & L PRECENTRAL GYRUS & $r=-.453 ; p=.02 *(C l:-.699$ to -.017$)$ \\
\hline IMG- & CL7 & 47 & $-22-65-9$ & 4,15 & L LINGUAL GYRUS & ns \\
\hline IMG- & CL9 & 146 & -521221 & 4,13 & L INFERIOR FRONTAL GYRUS (p. opercularis) & ns \\
\hline IMG- & CL10 & 9 & 0350 & 4,07 & L ANTERIOR CINGULATE CORTEX & ns \\
\hline IMG- & CL11 & 41 & $-30-7036$ & 4,04 & L MIDDLE OCCIPITAL GYRUS & ns \\
\hline IMG- & CL12 & 8 & $-32-1545$ & 3,99 & L PRECENTRAL GYRUS & ns \\
\hline IMG- & CL13 & 19 & $-10-9015$ & 3,97 & L CUNEUS & ns \\
\hline IMG- & CL15 & 60 & 177730 & 3,86 & R CUNEUS & ns \\
\hline IMG- & CL16 & 6 & 155718 & 3,81 & R SUPERIOR MEDIAL FRONTAL GYRUS & ns \\
\hline IMG- & CL19 & 29 & $-50-5215$ & 3,70 & L MIDDLE TEMPORAL GYRUS & $r=.-476 ; p=.014 *(C l:-704$ to -215$)$ \\
\hline IMG- & CL20 & 6 & -304527 & 3,68 & L MIDDLE FRONTAL GYRUS & ns \\
\hline IMG- & CL21 & 13 & $-47-26$ & 3,67 & L ROLANDIC OPERCULUM & Ns \\
\hline IMG- & CL22 & 12 & $-60-453$ & 3,62 & L MIDDLE TEMPORAL GYRUS & Ns \\
\hline IMG- & CL24 & 9 & $-23-7518$ & 3,60 & L MIDDLE OCCIPITAL GYRUS & Ns \\
\hline IMG- & CL26 & 23 & $48-5315$ & 3,54 & R MIDDLE TEMPORAL GYRUS & Ns \\
\hline IMG- & CL27 & 19 & $2-4530$ & 3,54 & R POSTERIOR CINGULATE CORTEX & Ns \\
\hline IMG- & CL28 & 18 & $-2718-6$ & 3,51 & L INSULA & Ns \\
\hline IMG- & CL29 & 12 & -45409 & 3,44 & L INFERIOR FRONTAL GYRUS (p. triangularis) & Ns \\
\hline IMG- & CL30 & 9 & $27-7712$ & 3,42 & R MIDDLE OCCIPITAL GYRUS & ns \\
\hline IMG- & CL31 & 11 & $-32-4554$ & 3,40 & L INFERIOR PARIETAL LOBULE & Ns \\
\hline IMG- & CL32 & 10 & $57-3727$ & 3,39 & R SUPRAMARGINAL GYRUS & Ns \\
\hline IMG- & CL33 & 11 & $-5-720$ & 3,33 & L LINGUAL GYRUS & Ns \\
\hline IMG- & CL34 & 10 & $10-60-6$ & 3,33 & R LINGUAL GYRUS & Ns \\
\hline IMG- & CL35 & 9 & $25-47-15$ & 3,33 & R FUSIFORM GYRUS & Ns \\
\hline IMG- & CL37 & 9 & $-4535-6$ & 3,29 & L INFERIOR FRONTAL GYRUS (p. orbitalis) & \\
\hline IMG- & CL38 & 7 & $25-1560$ & 3,26 & R PRECENTRAL GYRUS & Ns \\
\hline IMG- & CL40 & 10 & $-10-5069$ & 3,20 & L PRECUNEUS & Ns \\
\hline IMG- & CL41 & 5 & $-15-7527$ & 3,17 & L SUPERIOR OCCIPITAL GYRUS & ns \\
\hline IMG & CL42 & 5 & $-52-500$ & 3,17 & L MIDDLE TEMPORAL GYRUS & ns \\
\hline IMG- & CL43 & 8 & $2-806$ & 3,13 & L CALCARINE GYRUS & ns \\
\hline IMG- & CL44 & 10 & $0-2251$ & 3,07 & R SMA & ns \\
\hline IMG- & CL45 & 6 & 0626 & 3,05 & L SUPERIOR MEDIAL GYRUS & ns \\
\hline IMG- & CL46 & 7 & $-10-600$ & 3,03 & L LINGUAL GYRUS & ns \\
\hline $\mathrm{CA}+$ & CL1 & 11 & $-205-9$ & 3,77 & L PUTAMEN & ns \\
\hline CA+ & CL2 & 6 & $-42-242$ & 3,76 & L POSTCENTRAL GYRUS & ns \\
\hline
\end{tabular}




\begin{tabular}{|c|c|c|c|c|c|c|}
\hline CA+ & CL3 & 7 & $37-2218$ & 3,57 & R ROLANDIC OPERCULUM & ns \\
\hline $\mathrm{CA}+$ & CL4 & 8 & $10-37-12$ & 3,52 & R CEREBELLUM & ns \\
\hline CA+ & CL5 & 8 & -10150 & 3,19 & L CAUDATE & ns \\
\hline CA- & CL1 & 24 & $25-6015$ & 5,06 & R CALCARINE GYRUS & ns \\
\hline CA- & CL4 & 132 & -402227 & 4,20 & L INFERIOR FRONTAL GYRUS (p. triangularis) & ns \\
\hline CA- & CL7 & 11 & 42320 & 3,91 & R INFERIOR FRONTAL GYRUS (p. triangularis) & ns \\
\hline CA- & CL9 & 13 & $5-8530$ & 3,81 & L CUNEUS & ns \\
\hline CA- & CL14 & 6 & $-52-520$ & 3,54 & L MIDDLE TEMPORAL GYRUS & ns \\
\hline CA- & CL15 & 15 & $-50-20-12$ & 3,49 & L MIDDLE TEMPORAL GYRUS & ns \\
\hline CA- & CL17 & 8 & 20529 & 3,29 & R SUPERIOR FRONTAL GYRUS & ns \\
\hline CA- & CL19 & 7 & $-45-4527$ & 3,24 & L SUPRAMARGINAL GYRUS & ns \\
\hline CA- & CL20 & 5 & $-30-7039$ & 3,23 & L MIDDLE OCCIPITAL GYRUS & ns \\
\hline CA- & CL24 & 12 & $-10-9012$ & 3,10 & L CALCARINE GYRUS & ns \\
\hline CA- & CL25 & 10 & $-2727-6$ & 3,06 & L INFERIOR FRONTAL GYRUS (p. orbitalis) & ns \\
\hline
\end{tabular}




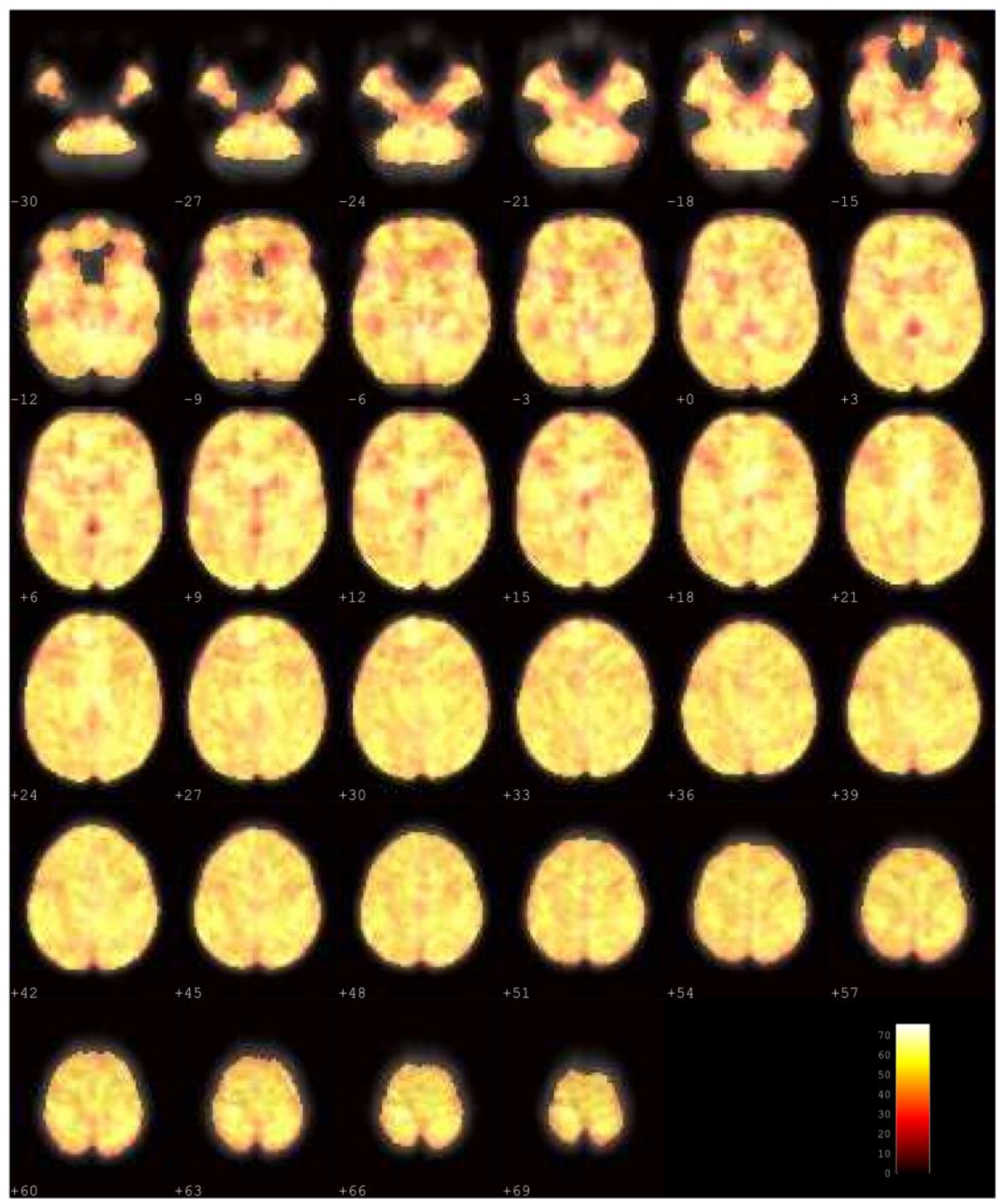

Supplementary Figure 1. Temporal signal-to-noise ratio (TSNR) average map showing EPI image quality over the whole brain. The color gradient indicates the mean TSNR of the smoothed EPI time course data (average of all images ( $n=258$ images $x 2$ runs $=516$ images) for each participant $(n=26)$ overlaid on the mean of the smoothed EPI time course data (average of smoothed EPI images for all participants ( $n=13416$ images). TSNR was calculated by dividing the mean signal intensity at a voxel by the standard deviation of its signal time course. The color map ranges from 0 (areas in black) to a TSNR of 75 (areas in white). 


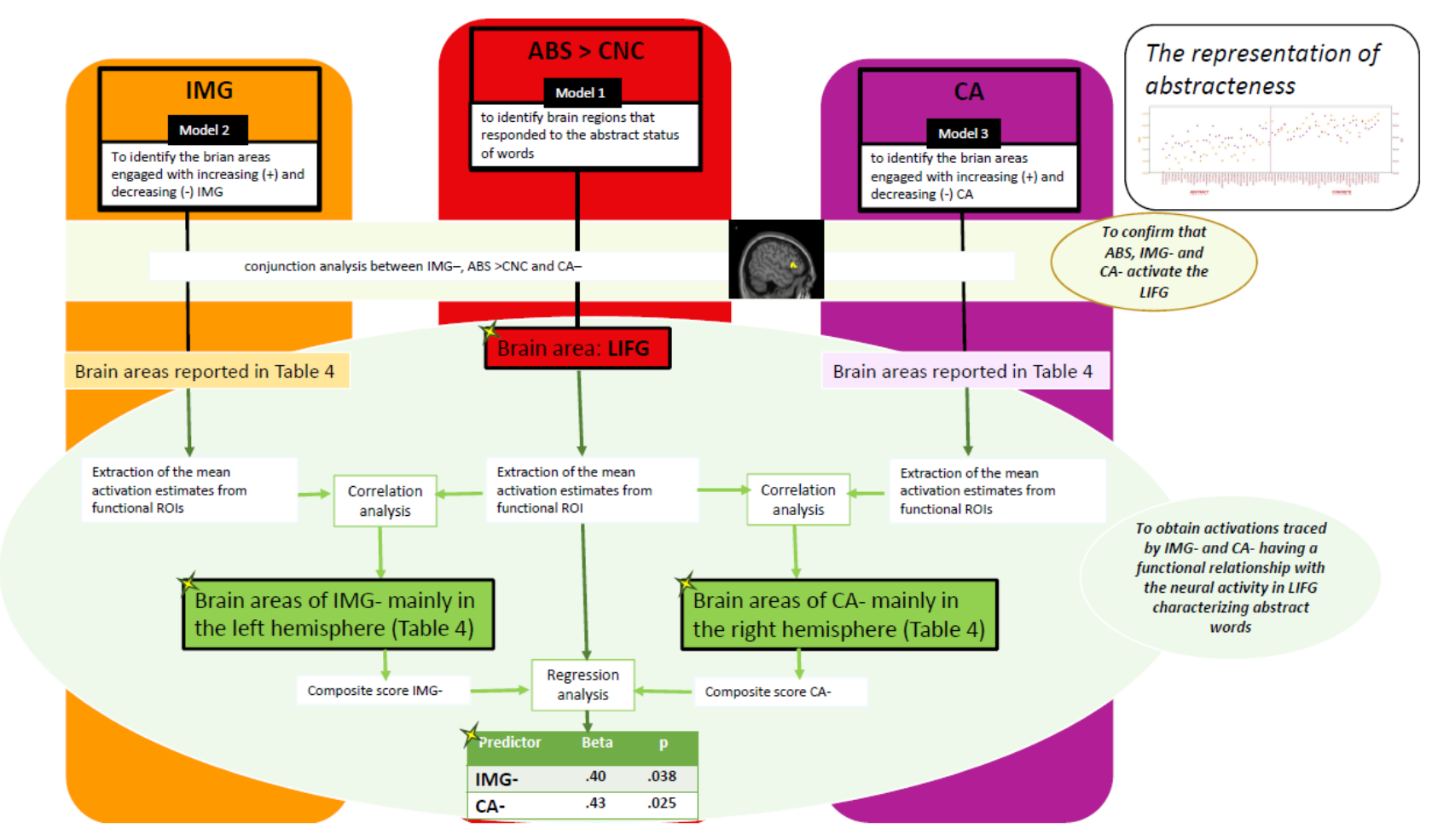

Supplementary Figure 2: Flow chart illustrating the logical flow and the steps performed for the representation of abstractness methodological section.

1) Orange, red and purple rectangles - The three rectangles represent the first three independent GLM models (Model 1: red, Model 2: orange, and Model 3: purple) performed in order to identify:

a. Model 1: the brain areas responding to abstractness more than concreteness (i.e. Abstract > concrete - red).

b. Models 2 and 3: the brain areas which respond to two constructs which are both representations of abstractness, though conveying different types of information concerning more intrinsic properties of the stimuli (IMG - orange), and more extrinsic properties (CA purple).

2) Light yellow rectangle - To confirm that ABS, IMG- and CA- show overlapping activity in the LIFG a conjunction analysis was carried out. The conjunction analysis is the neural starting point from which we want to investigate different functional associations (i.e. the representation of abstractness) and connectivity strength between areas (i.e. the processing of abstractness) in the activity pattern for IMG or CA with a shared LIFG activation between Abstract, less Imageable and less contextually available words.

3) Light green oval - The aim was to highlight which brain areas specific to IMG and CA show a significant and specific functional association with the neural timeseries in LIFG characterizing the abstract status of words. We investigated the pattern of activation in IMG and CA statistical maps (i.e. the activation clusters for increases or decreases of each construct reported in Table 4, see respectively the light orange and light pink rectangles). Mean activation estimates were extracted from functional ROIs built on clusters reported in Table 4, namely for both increases and decreases of IMG and CA (respectively model 1 and model 2) and from the LIFG cluster of activity, resulting from abstract > concrete constrast (model 1). Correlations between mean activation estimates of the brain areas associated to IMG (on the left), CA (on the right) and of the LIFG (resulting from model 1) were performed. Results are reported in the green rectangles: left brain areas for IMG- and right brain regions for CA-. These brain areas (separately for IMG- and for CA-) were used to derive two composite scores, one for IMG- and one for CA-. Both composite scores were used to predict the 
mean activation estimates of the LIFG (of model 1). Both predictors resulted significant. For further details, please see text.

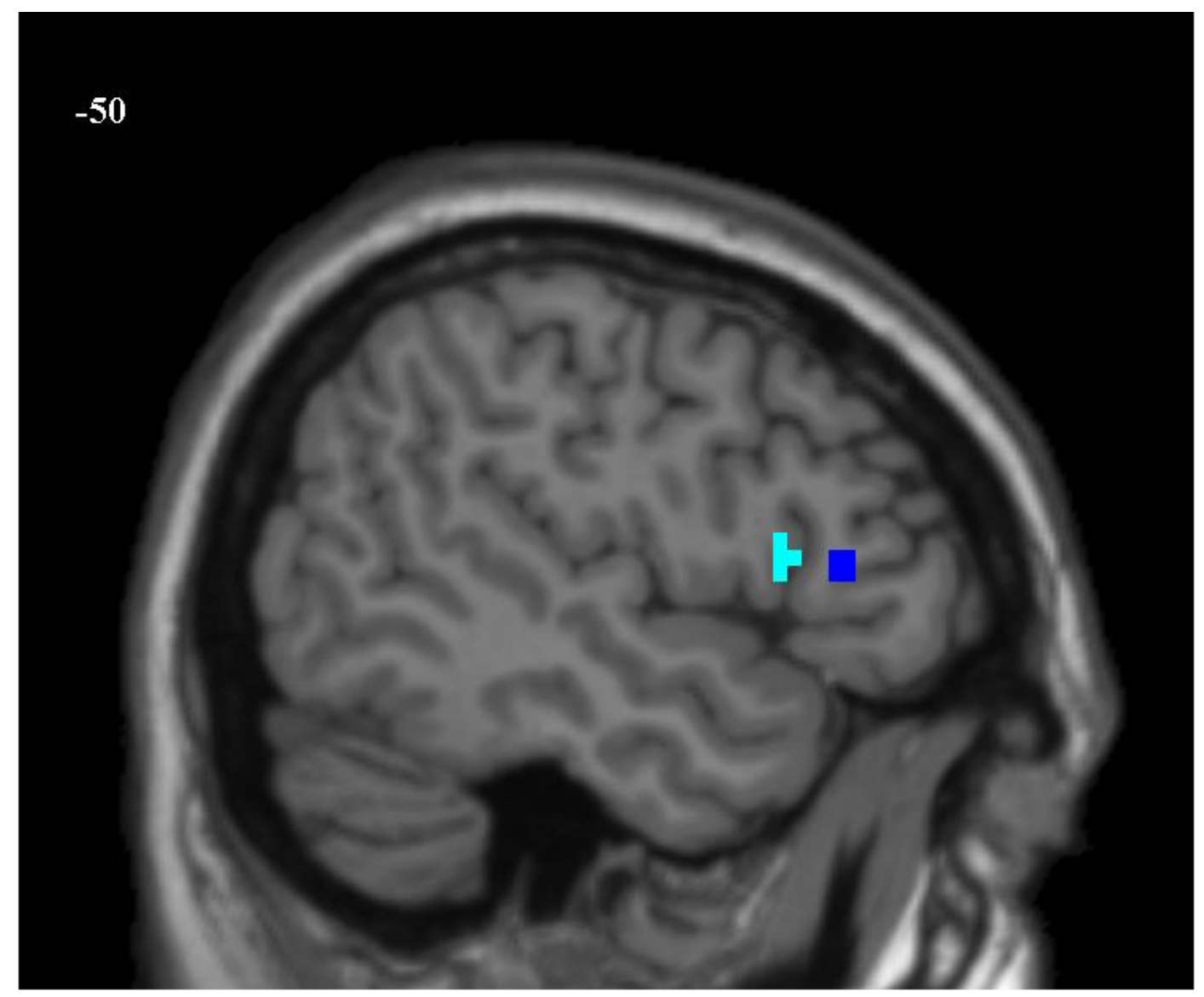

Supplementary Figure 3: The overlay of the LIFG clusters resulting from the "context-availability residual model" associated to decreases of context-availability thresholded at $p=.05$ (FWE-corrected) at the voxel level on a sagittal slice of standard brain (i.e. the MNI-152 template) with FIVE (http://mrtools.mgh.harvard.edu/index.php?title=Main Page). (blue: $x=-50, y=28, z=6 ; k=6$ voxels - BA 45 pars trinagularis; cyan: $x=-50, y=15, z=9 ; k=5$ voxels - BA 44 pars opercularis). 


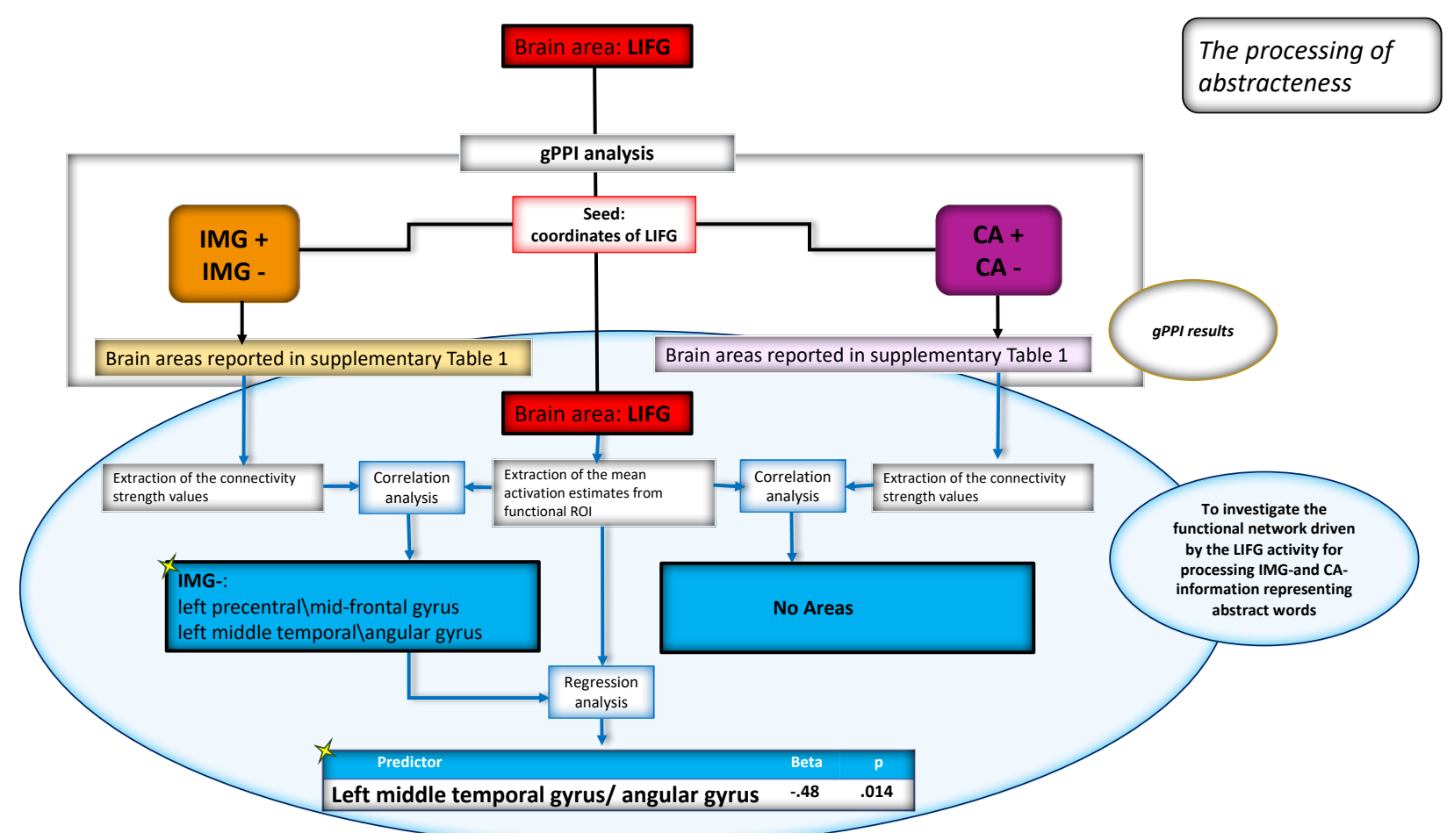

Supplementary Figure 4: Flow chart illustrating the logical flow and the steps performed for the processing of abstractness methodological section.

1) White rectangle: gPPI analysis - The aim of the gPPI analysis was to identify a 'source' region (i.e. the LIFG functional ROI resulting from the main effect of Abstract > Concrete in Model 1) and identify any other 'target' voxels/clusters in the brain with which that source has context-dependent connectivity. Target regions need not to correlate with the task or context alone (i.e. associations between functional IMG and CA ROIs and the LIFG functional timeseries as in the representation of abstractness section), but the interactions between these factors. Functional connectivity between LIFG seed region from model 1 (abstract>concrete, see Flow Chart 1), and whole brain during modulation of IMG (+ and -) and CA (+ and -) has been performed. Brain areas functionally connnected with the LIFG for IMG and CA were reported in the supplementary Table 1.

2) Light blue Oval: Connectivity strength values were extracted from the brain areas reported in supplementary Table 1, namely for both IMG and CA. Correlations between connectivity strength values of IMG (on the left), of CA (on the right) and the mean activation estimates extracted from the LIFG (resulting from model 1, red rectangle, see flow chart I) were performed. Results are reported in the blue rectangles. Only the left precentral \mid-frontal gyrus and left middle temporal \angular gyrus associated with IMG- resulted significantly correlated with the activation of the LIFG. A regression analysis showed that only the left middle temporal $\backslash$ angular gyrus was a significant predictor of the LIFG activation. 